\title{
A study of defect-based error estimates for the Krylov approximation of $\varphi$-functions
}

\section{Tobias Jawecki ${ }^{1}$ (iD}

Received: 31 January 2020 / Accepted: 17 August 2021/Published online: 8 November 2021

(C) The Author(s) 2021

\begin{abstract}
Prior recent work, devoted to the study of polynomial Krylov techniques for the approximation of the action of the matrix exponential $\mathrm{e}^{t A} v$, is extended to the case of associated $\varphi$-functions (which occur within the class of exponential integrators). In particular, a posteriori error bounds and estimates, based on the notion of the defect (residual) of the Krylov approximation are considered. Computable error bounds and estimates are discussed and analyzed. This includes a new error bound which favorably compares to existing error bounds in specific cases. The accuracy of various error bounds is characterized in relation to corresponding Ritz values of $A$. Ritz values yield properties of the spectrum of $A$ (specific properties are known a priori, e.g., for Hermitian or skew-Hermitian matrices) in relation to the actual starting vector $v$ and can be computed. This gives theoretical results together with criteria to quantify the achieved accuracy on the fly. For other existing error estimates, the reliability and performance are studied by similar techniques. Effects of finite precision (floating point arithmetic) are also taken into account.
\end{abstract}

Keywords Matrix exponential $\cdot \varphi$-functions $\cdot$ Krylov approximation · Upper bound $\cdot$ A posteriori error estimation

Mathematics Subject Classification (2010) $15 \mathrm{~A} 16 \cdot 65 \mathrm{~F} 60 \cdot 65 \mathrm{~L} 70 \cdot 65 \mathrm{~N} 22$

\section{Introduction}

Overview on prior work The matrix exponential and associated $\varphi$-functions play a crucial role in some numerical methods for solving systems of differential equations. In practice, this means that the vector $\mathrm{e}^{t A} v$ for a time step $t$, for a given matrix $A$

Tobias Jawecki

tobias.jawecki@asc.tuwien.ac.at

1 Institut für Analysis und Scientific Computing, Technische Universität Wien, Wiedner Hauptstrasse 8-10/E101, A-1040 Vienna, Austria 
and a given vector $v$, representing the time propagation for a linear initial value problem, is to be approximated. Similarly, the associated $\varphi$-functions (see (2.2) below) conform to solutions of certain inhomogeneous differential equations. In particular, evaluation of $\varphi$-functions is used in exponential integrators [27].

If the matrix $A$ is sparse and large, approximation of the action of these matrix functions in the class of Krylov subspaces is a general and well-established technique. For the matrix exponential and $\varphi$-functions, this goes back to early works in the field of chemical physics [39,44], parabolic problems [20], some nonlinear problems [18], etc. The case of a symmetric or skew-Hermitian matrix $A$ is the most prominent one. Krylov approximations of the matrix exponential were early studied for the symmetric case in $[12,13,46]$, and together with $\varphi$-functions in a more general setting [26, 28].

Concerning different approaches for the numerical approximation of the matrix exponential see [36]. In [46] it is shown for the symmetric case that the Krylov approximation is equivalent to interpolation of the exponential function at associated Ritz values. This automatically results in a near-best approximation among other choices of interpolation nodes, see also [12, 52] and further works [3] with similar results for the non-symmetric case and general functions including $\varphi$-functions. For other polynomial approaches approximating the matrix exponential, we mention truncated Taylor series [2] (and many works well in advance), Chebychev interpolation [54], or the Leja method [8], where [2] also covers $\varphi$-functions.

In general, Krylov approximations (or other polynomial approximations) result in an accurate approximation if the time step $t$ in $\mathrm{e}^{t A} v$ is sufficiently small or the dimension of the Krylov subspace (i.e., the degree of the approximating matrix polynomial) is sufficiently large, see for instance [26]. The dimension of the Krylov subspace is limited in practice, and large time steps require a restart of the iteration generating the Krylov basis. A larger time step $t$ can be split into smaller substeps for which the Krylov approximation can be applied in a nested way. Such a restarting strategy in the sense of a time integrator was already exploited in [44]. In particular we refer to the EXPOKIT package [49]. Similar ideas can be applied for the evaluation of $\varphi$-functions [28, 41, 49].

In practice, a posteriori error estimates are used to choose a proper Krylov dimension or proper (adaptive) substeps if the method is restarted as a time integrator. Different approaches for a posteriori error estimation concerning the exponential function make use of a series expansion for the error given [46] or use a formulation via the defect (also called residual) of the Krylov approximation [5, 9, 11, 28]. A prominent error estimate concerning $\varphi$-functions is the generalized residual estimate introduced in [28], which is based on the residual of a matrix inverse. Furthermore, a series expansion of the error concerning $\varphi$-functions is given in [49] (similar to the series expansion concerning the exponential in [46]) and leading terms of this series are used for a posteriori error estimation in [41, 49]. Further a priori as well as a posteriori error estimates for the exponential function are are given in $[3,10$, $30,31,34,37,56]$, where [10, 30] also consider $\varphi$-functions. Restarting via substeps based on different choices of error estimates is further discussed in [30]. A restart with substeps together with a strategy to choose the Krylov dimension in terms of 
computational cost was presented in [6, 41]. For various other approaches for restarting (without adapting the time step) we refer to [1, 5, 9, 15, 16, 40, 48, 53].

The influence of round-off errors on the construction of the Krylov basis in floating point arithmetic was early studied for the symmetric case in [43, 45]. The orthogonalization procedure can behave numerically unstable, typically due to a loss of orthogonality. Nevertheless, the near-best approximation property and related a priori convergence results are not critically affected [11, 13]. Following [11], in the symmetric case the defect obtained in floating point arithmetic results in numerically stable error estimates.

Beside the polynomial Krylov method, further studies are devoted to the approximation of matrix functions using so called extended Krylov subspaces [14, 21, 32], rational Krylov subspaces [17, 22, 38], or polynomial Krylov subspaces with a harmonic Ritz approach [25, 48, 57].

Overview on results presented here In Section 2, we introduce the problem setting and recapitulate basic properties of Krylov subspaces.

In Section 3, we introduce the defect associated with Krylov approximations to $\varphi$ functions, including the exponential function as the basic case. Our approach for the defect is different from [57] and is based on an inhomogeneous differential equation for the approximation error. This is used in Theorem 1 to obtain an integral representation of the error, also taking effects of floating point arithmetic into account. ${ }^{1}$ In contrast to previous works $([11,30])$, this result is extended to $\varphi$-functions here.

This upper bound is further analyzed in Section 4 to obtain computable a posteriori bounds, in particular a new a posteriori bound (Theorem 4). We also study the accuracy of our and other existing defect-based bounds [30] with respect to spectral properties of the Krylov Hessenberg matrix (the representation of $A$ in the orthogonal Krylov basis). To this end we use properties of divided differences including a new asymptotic expansion for these given in Appendix C. In Section 4.1, we consider error estimates based on a quadrature estimate of the defect norm integral: The generalized residual estimate [28] for the approximation of $\varphi$-functions which conforms to a quadrature of the defect norm integral (namely, the right-endpoint rectangle rule), and the effective order estimate, which was introduced for the approximation of the matrix exponential in [30] and is extended to $\varphi$-functions in the present work. We also discuss cases for which the defect norm behaves oscillatory and reliable quadrature estimates may be difficult to obtain. In Section 4.2, we specify a stopping criterion for the so-called lucky breakdown in floating point arithmetic which is justified by our a posteriori error bounds.

In Section 5, we illustrate our results via numerical experiments. This includes further remarks on previously known error estimates for the Krylov approximation of $\varphi$-functions.

\footnotetext{
${ }^{1} \mathrm{Cf}$. [11] for the case of the matrix exponential.
} 


\section{Problem statement and Krylov approximation}

We discuss the approximation via Krylov techniques for evaluation of the matrix exponential, and in particular of the associated $\varphi$-functions, for a step size $t>0$ and matrix $A \in \mathbb{C}^{n \times n}$ applied to an initial vector $v \in \mathbb{C}^{n}$. Here,

$$
\mathrm{e}^{t A} v=\sum_{k=0}^{\infty} \frac{(t A)^{k}}{k !} v \text {. }
$$

The matrix exponential $u(t)=\mathrm{e}^{t A} v$ is the solution of the differential equation

$$
u^{\prime}(t)=A u(t), \quad u(0)=v .
$$

The associated $\varphi$-functions are given by

$$
\varphi_{p}(t A) v=\sum_{k=0}^{\infty} \frac{(t A)^{k}}{(k+p) !} v, \quad p \in \mathbb{N}_{0} .
$$

This includes the case $\varphi_{0}=$ exp. The matrix functions (2.1) and (2.2) are defined according to their scalar counterparts. The following definitions of $\varphi_{p}$ are equivalent to (2.2): For $z \in \mathbb{C}$ we have $\varphi_{0}(z)=\mathrm{e}^{z}$, and

$$
\varphi_{p}(z)=\frac{1}{(p-1) !} \int_{0}^{1} \mathrm{e}^{(1-\theta) z} \theta^{p-1} \mathrm{~d} \theta, \quad p \in \mathbb{N} .
$$

(See also [24, Section 10.7.4].) The function $w_{p}(t)=t^{p} \varphi_{p}(t A) v(p \in \mathbb{N})$ is the solution of an inhomogeneous differential equation of the form

$$
w_{p}^{\prime}(t)=A w_{p}(t)+\frac{t^{p-1}}{(p-1) !} v, \quad w_{p}(0)=0,
$$

see for instance [41]. This follows from (2.2),

$$
\begin{aligned}
\frac{\mathrm{d}}{\mathrm{d} t}\left(t^{p} \varphi_{p}(t A) v\right) & =\frac{\mathrm{d}}{\mathrm{d} t}\left(\sum_{k=0}^{\infty} \frac{t^{k+p} A^{k} v}{(k+p) !}\right)=A \sum_{k=0}^{\infty} \frac{t^{k+p} A^{k} v}{(k+p) !}+\frac{t^{p-1} v}{(p-1) !} \\
& =A\left(t^{p} \varphi_{p}(t A) v\right)+\frac{t^{p-1} v}{(p-1) !} .
\end{aligned}
$$

The $\varphi$-functions appear for instance in the field of exponential integrators, see for instance [27].

For the case of $A$ being a large and sparse matrix, e.g., the spatial discretization of a partial differential operator using a localized basis, Krylov subspace techniques are commonly used to approximate (2.2) in an efficient way.

Notation and properties of Krylov subspaces ${ }^{2}$ We briefly recapitulate the usual notation and properties of standard Krylov subspaces, see for instance [47]. For a given

\footnotetext{
${ }^{2}$ In the sequel, $e_{j}$ denotes the $j$ th unit vector in $\mathbb{C}^{m}$ or $\mathbb{C}^{n}$, respectively.
} 
matrix $A \in \mathbb{C}^{n \times n}$, a starting vector $v \in \mathbb{C}^{n}$ and Krylov dimension $0<m \leq n$, the Krylov subspace is given by

$$
\mathscr{K}_{m}(A, v)=\operatorname{span}\left(v, A v, \ldots, A^{m-1} v\right) .
$$

Let $V_{m} \in \mathbb{C}^{n \times m}$ represent the orthonormal basis of $\mathscr{K}_{m}(A, v)$ with respect to the Hermitian inner product, constructed by the Arnoldi method and satisfying $V_{m}^{*} V_{m}=$ $I_{m \times m}$. Its first column is given by $V_{m}^{*} v=\beta e_{1}$ with $\beta=\|v\|_{2}$. Here, the matrix

$$
H_{m}=V_{m}^{*} A V_{m} \in \mathbb{C}^{m \times m}
$$

is upper Hessenberg. We further use the notation $h_{m+1, m}=\left(H_{m+1}\right)_{m+1, m} \in \mathbb{R}$, and $v_{m+1} \in \mathbb{C}^{n}$ for the $(m+1)$ th column of $V_{m+1}$, with $V_{m}^{*} v_{m+1}=0$ and $\left\|v_{m+1}\right\|_{2}=1$.

The Arnoldi decomposition (in exact arithmetic) can be expressed in matrix form,

$$
A V_{m}=V_{m} H_{m}+h_{m+1, m} v_{m+1} e_{m}^{*} .
$$

Remark 1 The numerical range $\mathrm{W}(A)=\left\{y^{*} A y / y^{*} y, \quad 0 \neq y \in \mathbb{C}^{n}\right\}$ plays a role in our analysis. Note that $\mathrm{W}\left(H_{m}\right) \subseteq \mathrm{W}(A)$ (see (A.1)).

Remark 2 The case $\left(H_{m}\right)_{k+1, k}=0$ occurs if $\mathscr{K}_{k}(A, v)$ is an invariant subspace of $A$, whence the Krylov approximation given in (2.9) below is exact. This exceptional case is referred to as a lucky breakdown. In general, we assume that no lucky breakdown occurs, whence the lower subdiagonal entries of $H_{m}$ are real and positive, $0<\left(H_{m}\right)_{j+1, j}$ for $j=1, \ldots, m-1$, and $0<h_{m+1, m} \in \mathbb{R}$.

For the special case of a Hermitian or skew-Hermitian matrix $A$ the Arnoldi iteration simplifies to a three-term recurrence, the so-called Lanczos iteration. This case will be addressed in Remark 4 below.

Krylov subspaces in floating point arithmetic We proceed with some results for the Arnoldi decomposition in computer arithmetic, assuming complex floating point arithmetic with a relative machine precision $\varepsilon$, see also [23]. For practical implementation different variants of the Arnoldi procedure exist, using different ways for the orthogonalization of the Krylov basis. These are based on classical Gram-Schmidt, modified Gram-Schmidt, the Householder algorithm, the Givens algorithm, or variants of Gram-Schmidt with reorthogonalization (see also [47, Algorithm 6.1-6.3] and others). We refer to [7] and references therein for an overview on the stability properties of these different variants.

In the sequel, the notation $V_{m}, H_{m}$, etc., will again be used for the result of the Arnoldi method in floating point arithmetic. We now accordingly adapt some statements formulated in the previous paragraph. By construction, $H_{m}$ remains to be upper Hessenberg with positive lower subdiagonal entries. Assuming floating point arithmetic, we use the notation $U_{m} \in \mathbb{C}^{n \times m}$ for a perturbation of the Arnoldi decomposition (2.5) caused by round-off, i.e.,

$$
A V_{m}=V_{m} H_{m}+h_{m+1, m} v_{m+1} e_{m}^{*}+U_{m} .
$$

An upper norm bound for $U_{m}$ was first introduced in [43] for the Lanczos iteration in real arithmetic. For different variants of the Arnoldi or Lanczos iteration, this is 
discussed in [58] and others. We assume $\left\|U_{m}\right\|_{2}$ is bounded by a constant $C_{1}$ which can depend on $m$ and $n$ in a moderate way and is sufficiently small in a typical setting,

$$
\left\|U_{m}\right\|_{2} \leq C_{1} \varepsilon\|A\|_{2} \text {. }
$$

We further assume that the normalization of the columns of $V_{m}$ is accurate, in particular that the $(m+1)$ th basis vector $v_{m+1}$ is normalized correctly up round-off with a sufficiently small constant $C_{2}$ (see e.g., [43, (14)]),

$$
\left|\left\|v_{m+1}\right\|_{2}-1\right| \leq C_{2} \varepsilon \text {. }
$$

Concerning $V_{m+1}$ which represents an orthogonal basis in exact arithmetic, numerical loss of orthogonality has been well-studied. Loss of orthogonality can be significant (see for instance [7, 45] and others), depending on the starting vector $v$. Reorthogonalization schemes or orthogonalization via Householder or Givens algorithm can be used to obtain orthogonality of $V_{m+1}$ on a sufficiently accurate level.

The numerical range of $H_{m}$ obtained in floating point arithmetic (see (2.6)) can be characterized as

$$
\mathrm{W}\left(H_{m}\right) \subseteq U_{C_{3} \varepsilon}(\mathrm{W}(A)),
$$

with $U_{C_{3} \varepsilon}(\mathrm{W}(A))$ being the neighborhood of $\mathrm{W}(A)$ in $\mathbb{C}$ with a distance $C_{3} \varepsilon$. With the assumption that $V_{m+1}$ is sufficiently close to orthogonal (e.g., semiorthogonal [50]), the constant $C_{3}$ in $(2.7 \mathrm{c})$ (which also depends on $C_{1}$ and problem sizes) can be shown to be moderate-sized. Further details on this aspect are given in Appendix A.

Krylov approximation of $\boldsymbol{\varphi}$-functions ${ }^{3}$ Let $V_{m} \in \mathbb{C}^{n \times m}, H_{m} \in \mathbb{C}^{m \times m}$ and $\beta \in \mathbb{R}$ be the result of the Arnoldi method in floating point arithmetic for $\mathscr{K}_{m}(A, v)$ as described above. For a time-step $0<t \in \mathbb{R}$ and $p \geq 0$, the vector $\varphi_{p}(t A) v$ can be approximated in the Krylov subspace $\mathscr{K}_{m}(A, v)$ by the Krylov propagator

$$
u_{p, m}(t):=V_{m} \varphi_{p}\left(t V_{m}^{*} A V_{m}\right) V_{m}^{*} v=\beta V_{m} \varphi_{p}\left(t H_{m}\right) e_{1}, \quad p \in \mathbb{N} .
$$

The special case $p=0$ reads

$$
u_{0, m}(t)=\beta V_{m} \mathrm{e}^{t H_{m}} e_{1} .
$$

We remark that the small-dimensional problem $\varphi_{p}\left(t H_{m}\right) e_{1} \in \mathbb{C}^{m}$, typically with $m \ll n$, can be evaluated cheaply by standard methods. In the sequel, we denote

$$
y_{p, m}(t)=\beta \varphi_{p}\left(t H_{m}\right) e_{1} \in \mathbb{C}^{m}, \quad \text { i.e., } \quad u_{p, m}(t)=V_{m} y_{p, m}(t) .
$$

For $p=0$, the small dimensional problem $y_{0, m}(t)=\beta \mathrm{e}^{t H_{m}} e_{1}$ solves the differential equation

$$
y_{0, m}^{\prime}(t)=H_{m} y_{0, m}(t), \quad y_{0, m}(0)=\beta e_{1} .
$$

For later use, we introduce the notation

$$
\widehat{y}_{p, m}(t)=t^{p} y_{p, m}(t)
$$

\footnotetext{
${ }^{3}$ Remark concerning notation: " $u$ " objects live in $\mathbb{C}^{n}$, and " $y$ " objects live in $\mathbb{C}^{m}$.
} 
which for $p \in \mathbb{N}$ and according to (2.4) satisfies the differential equation

$$
\widehat{y}_{p, m}^{\prime}(t)=H_{m} \widehat{y}_{p, m}(t)+\frac{t^{p-1}}{(p-1) !} \beta e_{1}, \quad \widehat{y}_{p, m}(0)=0 .
$$

Remark 3 Although we take rounding effects in the Arnoldi decomposition into account, we do not give a full study of round-off errors at this point. Round-off errors in substeps such as the evaluation of $y_{p, m}(t)$ or the matrix-vector multiplication $V_{m} y_{p, m}(t)$ will be ignored. We refer to [23] for a more general study of these effects.

Remark 4 In the special cases $A=B$ or $A=\mathrm{i} B$ for a Hermitian matrix $B \in$ $\mathbb{C}^{n \times n}$ (with $A$ being skew-Hermitian in the latter case) the orthogonalization of the Krylov basis of $\mathscr{K}_{m}(B, v)$ simplifies to a three-term recursion, the so-called Lanczos method. In the skew-Hermitian case $(A=\mathrm{i} B)$ the Krylov propagator (2.8a) can be evaluated by $\beta V_{m} \varphi_{p}$ (it $\left.H_{m}\right) e_{1}$, i.e., we approximate the function $\lambda \mapsto \varphi_{p}(\mathrm{i} t \lambda)$ in the Krylov subspace $\mathscr{K}_{m}(B, v)$. The advantage is a cheaper computation of the Krylov subspace in terms of computational cost and better conservation of geometric properties. For details we refer to the notation $\mathrm{e}^{\sigma t B}$ as introduced in [30], with $\sigma= \pm \mathrm{i}$ and a Hermitian matrix $B$ for the skew-Hermitian case.

The error of the Krylov propagator We denote the error of the Krylov propagator given in (2.9) by

$$
l_{p, m}(t)=\beta V_{m} \varphi_{p}\left(t H_{m}\right) e_{1}-\varphi_{p}(t A) v, \quad p \in \mathbb{N}_{0} .
$$

We are further interested in computable a posteriori estimates for the error norm, $\zeta_{p, m}(t) \approx\left\|l_{p, m}(t)\right\|_{2}$, which in the best case can be proven to be upper bounds on the error norm $\left\|l_{p, m}(t)\right\|_{2} \leq \zeta_{p, m}(t)$. Norm estimates of the error (2.12) can be used in practice to stop the Krylov iteration after $k$ steps if $\left\|l_{p, k}(t)\right\|_{2}$ satisfies (2.13) below, or to restrict the time-step $t$ to obtain an accurate approximation and restart the method with the remaining time. For details on the total error with this restarting approach, see also [30, 49].

A prominent task is to test if the error norm per unit step is bounded by a tolerance tol,

$$
\zeta_{p, m}(t) \leq t \cdot \text { tol, } \quad \text { which should entail }\left\|l_{p, m}(t)\right\|_{2} \leq t \cdot \text { tol. }
$$

In case of $\zeta_{p, m}(t)$ being an upper bound on the error norm, this results in a reliable bound.

\section{An integral representation for the error of the Krylov propagator}

We proceed with discussing the error $l_{p, m}$ of the Krylov propagator. To this end, we first define its scalar defect by

$$
\delta_{p, m}(t)=\beta e_{m}^{*} t^{p} \varphi_{p}\left(t H_{m}\right) e_{1}=t^{p}\left(y_{p, m}(t)\right)_{m} \in \mathbb{C},
$$


and the defect integral by ${ }^{4}$

$$
L_{p, m}(t)=\frac{h_{m+1, m}}{t^{p}} \int_{0}^{t}\left|\delta_{p, m}(s)\right| \mathrm{d} s \in \mathbb{R} .
$$

Theorem 1 Let $\delta_{p, m}(t) \in \mathbb{C}$ be the defect defined in (3.1a). For $y_{p, m}(t) \in$ $\mathbb{C}^{m}$ defined in (2.9) and a numerical perturbation $U_{m} \in \mathbb{C}^{n \times m}$ of the Arnoldi decomposition (see (2.6)), we have:

(a) The error $l_{p, m}(t)$ of the Krylov propagator (see (2.12)) enjoys the integral representation

$l_{p, m}(t)=-\frac{h_{m+1, m}}{t^{p}} \int_{0}^{t} \mathrm{e}^{(t-s) A} v_{m+1} \delta_{p, m}(s) \mathrm{d} s-\frac{1}{t^{p}} \int_{0}^{t} \mathrm{e}^{(t-s) A} U_{m} s^{p} y_{p, m}(s) \mathrm{d} s$.

(b) For given machine precision $\varepsilon$ and constants $C_{1}, C_{2}$ representing roundoff effects (see (2.7a),(2.7b)), and with $\kappa_{1}=\max _{s \in[0, t]}\left\|\mathrm{e}^{s A}\right\|_{2}$ and $\kappa_{2}=$ $\max _{s \in[0, t]}\left\|\mathrm{e}^{s H_{m}}\right\|_{2}$ the error norm is bounded by

$$
\left\|l_{p, m}(t)\right\|_{2} \leq\left(1+C_{2} \varepsilon\right) \kappa_{1} L_{p, m}(t)+C_{1} \varepsilon\|A\|_{2} \frac{\beta \kappa_{1} \kappa_{2} t}{(p+1) !},
$$

with the defect integral $L_{p, m}(t)$ defined in (3.1b).

Proof (a) For the exact matrix function, we use the notation

$$
u_{p}(t)=\varphi_{p}(t A) v, \quad \text { and } \quad w_{p}(t)=t^{p} u_{p}(t)
$$

For the Krylov propagator, we denote

$$
u_{p, m}(t)=V_{m} y_{p, m}(t) \text { with } y_{p, m}(t)=\beta \varphi_{p}\left(t H_{m}\right) e_{1}
$$

(see (2.9)), and we also define

$w_{p, m}(t)=t^{p} u_{p, m}(t)=V_{m} \widehat{y}_{p, m}(t), \quad$ with $\widehat{y}_{p, m}(t)=t^{p} y_{p, m}(t)$ defined in (2.11a).-

For $p \in \mathbb{N}$, the functions $w_{p}(t)$ and $w_{p, m}(t)$ satisfy the differential equations (see (2.4), (2.11b))

$$
\begin{aligned}
& w_{p, m}^{\prime}(t)=V_{m} \widehat{y}_{p, m}^{\prime}(t)=V_{m}\left(H_{m} \widehat{y}_{p, m}(t)+\frac{t^{p-1}}{(p-1) !} \beta e_{1}\right) \\
& w_{p}^{\prime}(t)=A w_{p}(t)+\frac{t^{p-1}}{(p-1) !} v, \quad \text { and } \quad w_{p}(0)=w_{p, m}(0)=0
\end{aligned}
$$

\footnotetext{
4 This and the result of Theorem 1 remain valid for the case $t=0$.
} 
- For $p=0$, i.e., $w_{0}(t)=u_{0}(t)$ and $w_{0, m}(t)=V_{m} y_{0, m}(t)$, according to $(2.10)$, we have

$$
\begin{aligned}
& w_{0}^{\prime}(t)=A w_{0}(t), \quad w_{0, m}^{\prime}(t)=V_{m} H_{m} y_{0, m}(t), \\
& \text { and } \quad w_{0}(0)=v, \quad w_{0, m}(0)=\beta V_{m} e_{1}=v .
\end{aligned}
$$

Local error representation in terms of the defect We defined the re-scaled error

$$
\widehat{l}_{p, m}(t)=w_{p, m}(t)-w_{p}(t)=t^{p} l_{p, m}(t) .
$$

- For $p \in \mathbb{N}$, this satisfies

$$
\widehat{l}_{p, m}^{\prime}(t)=w_{p, m}^{\prime}(t)-w_{p}^{\prime}(t)=A \widehat{l}_{p, m}(t)+d_{p, m}(t), \quad \widehat{l}_{p, m}(0)=0,
$$

with the defect of $w_{p, m}(t)$ with respect to the differential (3.3),

$$
\begin{aligned}
d_{p, m}(t) & =w_{p, m}^{\prime}(t)-A w_{p, m}(t)-\frac{t^{p-1}}{(p-1) !} v \\
& =V_{m}\left(H_{m} \widehat{y}_{p, m}(t)+\frac{t^{p-1}}{(p-1) !} \beta e_{1}\right)-A V_{m} \widehat{y}_{p, m}(t)-\frac{t^{p-1}}{(p-1) !} v \\
& =\left(V_{m} H_{m}-A V_{m}\right) \widehat{y}_{p, m}(t)+\frac{t^{p-1}}{(p-1) !}\left(\beta V_{m} e_{1}-v\right) .
\end{aligned}
$$

Together with (2.6) and using of $\beta V_{m} e_{1}=v$, the defect can be written as

$$
d_{p, m}(t)=-h_{m+1, m}\left(e_{m}^{*} \widehat{y}_{p, m}(t)\right) v_{m+1}-U_{m} \widehat{y}_{p, m}(t) .
$$

- For $p=0$, in an analogous way, we obtain

$$
d_{0, m}(t)=-h_{m+1, m}\left(e_{m}^{*} y_{0, m}(t)\right) v_{m+1}-U_{m} y_{0, m}(t) .
$$

We conclude

$$
d_{p, m}(t)=-h_{m+1, m} \delta_{p, m}(t) v_{m+1}-t^{p} U_{m} y_{p, m}(t), \quad p \in \mathbb{N}_{0},
$$

with the scalar defect defined in (3.1a). Due to (3.4), we have

$$
\widehat{l}_{p, m}(t)=\int_{0}^{t} \mathrm{e}^{(t-s) A} d_{p, m}(s) \mathrm{d} s, \quad p \in \mathbb{N}_{0},
$$

and for $l_{p, m}(t)=t^{-p \widehat{l}_{p, m}}(t)$ together with (3.5) this implies (3.2a).

(b) With $\kappa_{1}=\max _{s \in[0, t]}\left\|\mathrm{e}^{s A}\right\|_{2},\left\|U_{m}\right\|_{2} \leq C_{1} \varepsilon\|A\|_{2}$ and $\left\|v_{m+1}\right\|_{2} \leq 1+C_{2} \varepsilon$, the representation (3.2a) implies the upper bound

$$
\begin{aligned}
\left\|l_{p, m}(t)\right\|_{2} \leq(1+ & \left.C_{2} \varepsilon\right) \kappa_{1} \frac{h_{m+1, m}}{t^{p}} \int_{0}^{t}\left|\delta_{p, m}(s)\right| \mathrm{d} s \\
& +C_{1} \varepsilon\|A\|_{2} \frac{\kappa_{1}}{t^{p}} \int_{0}^{t} s^{p}\left\|y_{p, m}(s)\right\|_{2} \mathrm{~d} s .
\end{aligned}
$$


With the defect integral $L_{p, m}(t)$ defined in (3.1b) we obtain the first term in (3.2b). For the second integral term (with $\left.y_{p, m}(t)=\beta \varphi_{p}\left(t H_{m}\right) e_{1}\right)$, we use the upper bound

$$
\int_{0}^{t} s^{p}\left\|\varphi_{p}\left(s H_{m}\right) e_{1}\right\|_{2} \mathrm{~d} s \leq \max _{s \in[0, t]}\left\|\varphi_{p}\left(s H_{m}\right) e_{1}\right\|_{2} \frac{t^{p+1}}{p+1} .
$$

- For $p \in \mathbb{N}$ we apply the integral representation due to (2.3) for $\varphi_{p}\left(t H_{m}\right) e_{1}$ to obtain the norm bound

$$
\max _{s \in[0, t]}\left\|\varphi_{p}\left(s H_{m}\right) e_{1}\right\|_{2} \leq \frac{\max _{s \in[0, t]}\left\|\mathrm{e}^{s H_{m}}\right\|_{2}}{(p-1) !} \int_{0}^{1} \theta^{p-1} \mathrm{~d} \theta=\frac{\max _{s \in[0, t]}\left\|\mathrm{e}^{s H_{m}}\right\|_{2}}{p !}
$$

- For $p=0$, we obtain (3.8) in a direct way.

Combining (3.7) with (3.8) and denoting $\kappa_{2}=\max _{s \in[0, t]}\left\|\mathrm{e}^{s H_{m}}\right\|_{2}$, we obtain

$$
\frac{\kappa_{1}}{t^{p}} \int_{0}^{t} s^{p}\left\|y_{p, m}(s)\right\|_{2} \mathrm{~d} s \leq \frac{\beta \kappa_{1} \kappa_{2} t}{(p+1) !} .
$$

Combining these estimates with (3.6), we conclude (3.2b).

Remark 5 The error norm of the Krylov propagator scales with $\kappa_{1}=$ $\max _{s \in[0, t]}\left\|\mathrm{e}^{s A}\right\|_{2}$ and $\kappa_{2}=\max _{s \in[0, t]}\left\|\mathrm{e}^{s H_{m}}\right\|_{2}$ in a natural way. ${ }^{5}$ It is well known that

$$
\begin{aligned}
\left\|\mathrm{e}^{t A}\right\|_{2} \leq & \mathrm{e}^{t \mu_{2}(A)} \text { with the logarithmic norm } \\
& \mu_{2}(A)=\max \{\operatorname{Re}(\mathrm{W}(A))\}=\max \left\{\operatorname{spec}\left(A+A^{*}\right) / 2\right\},
\end{aligned}
$$

see for instance [24, Theorem 10.11]. Problems with $\mu_{2}(A)>0$ can be arbitrary ill-conditioned and difficult to solve with proper accuracy. (For further results on the stability of the matrix exponential see also [36, 55].). We will not further discuss problems with $\mu_{2}(A)>0$ and assume $\mu_{2}(A) \leq 0$. We refer to the case $\mu_{2}(A) \leq 0$ as the dissipative case, with $\kappa_{1}=1$.

For the dissipative case with $\mu_{2}(A) \leq 0$, the error bound (3.2b) from Theorem 1 reads

$$
\left\|l_{p, m}(t)\right\|_{2} \leq\left(1+C_{2} \varepsilon\right) L_{p, m}(t)+C_{1} \varepsilon\|A\|_{2} \frac{\beta \kappa_{2} t}{(p+1) !} .
$$

The dissipative behavior of $\mathrm{e}^{t A}$ carries over to the Krylov propagator up to a perturbation which depends on round-off errors, including the loss of orthogonality of $V_{m}$. In terms of the numerical range $\mathrm{W}\left(H_{m}\right)$, with $\mathrm{W}\left(H_{m}\right) \subseteq U_{C_{3} \varepsilon}(\mathrm{W}(A))$, we have $\mu_{2}\left(H_{m}\right) \leq \mu_{2}(A)+C_{3} \varepsilon$, for a constant $C_{3} \varepsilon$ depending on round-off effects $(2.7 \mathrm{c})$. Thus, $\mu_{2}\left(H_{m}\right) \leq C_{3} \varepsilon$ and $\kappa_{2} \leq \mathrm{e}^{t C_{3} \varepsilon}$.

\footnotetext{
${ }^{5}$ Taking the maximum $\max _{s \in[0, t]}$ in the definition of $\kappa_{1}$ and $\kappa_{2}$ is necessary to cover the case $p>0$. For the special case $p=0$ the upper norm bound given in Theorem 1 can be adapted to scale with $\mathrm{e}^{t \mu_{2}(A)}$.
} 
Our aim is to construct an upper norm bound for the error per unit step (2.13) via (3.9). Let the tolerance tol be given and $t$ be a respective time step for (2.13). Then the round-off error terms in (3.9) are negligible if

$$
C_{2} \varepsilon \ll 1, \text { and } C_{1} \varepsilon\|A\|_{2} \beta \mathrm{e}^{t C_{3} \varepsilon} /(p+1) ! \ll \text { tol. }
$$

Concerning the constants $C_{1}, C_{2}$ and $C_{3}$ see (2.7). We recapitulate that $C_{1}$ and $C_{2}$ given in (2.7a) and (2.7b) can be considered to be small enough in a standard Krylov setting. The constant $C_{3}$ can be larger in the case of a loss of orthogonality of the Krylov subspace, which can however be avoided at the cost of additional computational effort. The constant $C_{3}$ only appears as an exponential prefactor for the round-off term in (3.10) and is less critical compared to $C_{1}$ and $C_{2}$.

With the previous observation on the round-off errors taken into account in (3.9) we consider the following upper bound to be stable in computer arithmetic in accordance to a proper value of tol, see (3.10).

Corollary 1 For the case $\mu_{2}(A) \leq 0$ and with the assumption that round-off error is negligible, the error of the Krylov propagator is bounded by the defect integral $L_{p, m}(t)$,

$$
\left\|l_{p, m}(t)\right\|_{2} \leq \frac{h_{m+1, m}}{t^{p}} \int_{0}^{t}\left|\delta_{p, m}(s)\right| \mathrm{d} s=L_{p, m}(t), \quad p \in \mathbb{N}_{0} .
$$

Note that the defect norm $\left|\delta_{p, m}(s)\right|$ cannot be integrated exactly in general. This point will further be studied in the sequel.

Representing the defect in terms of divided differences Divided differences play an essential role in this work. We use the notation

$$
f\left[\lambda_{1}, \ldots, \lambda_{m}\right]
$$

for the divided differences of a function $f$ over the nodes $\lambda_{1}, \ldots, \lambda_{m}$. (This is to be understood in the confluent sense for the case of multiple nodes $\lambda_{j}$, see for instance [24, Section B.16].)

Theorem 2 (see for instance [9]) Let $H_{m} \in \mathbb{C}^{m \times m}$ be an upper Hessenberg matrix with positive secondary diagonal entries, $0<\left(H_{m}\right)_{j+1, j} \in \mathbb{R}$ for $j=1, \ldots, m-1$, and eigenvalues $\lambda_{1}, \ldots, \lambda_{m}$. Let $f$ be an analytic function for which $f\left(H_{m}\right)$ is well defined. Then,

$$
e_{m}^{*} f\left(H_{m}\right) e_{1}=\gamma_{m} f\left[\lambda_{1}, \ldots, \lambda_{m}\right],
$$

with $\gamma_{m}=\prod_{j=1}^{m-1}\left(H_{m}\right)_{j+1, j}$.

For $f=\left(\varphi_{p}\right)_{t}: \lambda \mapsto \varphi_{p}(t \lambda)$, we will also make use of the following result. ${ }^{6}$

\footnotetext{
${ }^{6}$ Theorem 3 can be generalized to the case $t^{p} e_{m}^{*} \varphi_{k+p}\left(t H_{m}\right) e_{1}=e_{m+p}^{*} \varphi_{k}\left(t \widetilde{H}_{p, m}\right) e_{1}$ with $k \in \mathbb{N}$, see [2, Theorem 2.1]. The case $k=0$ is sufficient for our purpose.
} 
Theorem 3 (Corollary 1 in [49]) (Expressing $\varphi$-functions via dilated exp-functions.) For $t \in \mathbb{R}$,

$$
t^{p} e_{m}^{*} \varphi_{p}\left(t H_{m}\right) e_{1}=e_{m+p}^{*} \exp \left(t \widetilde{H}_{p, m}\right) e_{1}
$$

with

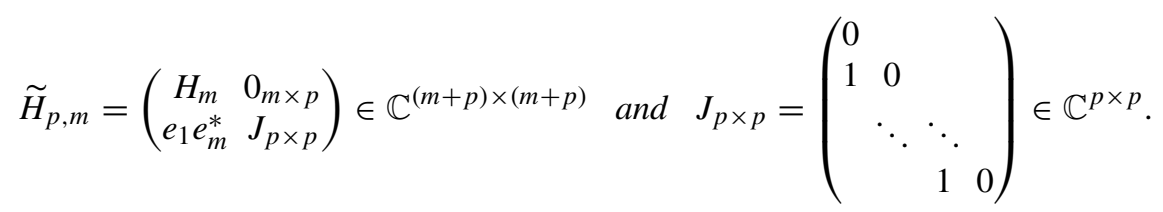

The matrix $\widetilde{H}_{p, m}$ in Theorem 3 is block triangular with eigenvalues equal to those of $H_{m}$ and $J_{p \times p}$. Therefore, $\operatorname{spec}\left(\widetilde{H}_{m}\right)=\left\{\lambda_{1}, \ldots, \lambda_{m}, 0, \ldots, 0\right\}$, with 0 as an eigenvalue of multiplicity $p$ (at least). In our context, $\widetilde{H}_{m}$ is upper Hessenberg with a positive lower secondary diagonal and $\gamma_{m}=\prod_{j=1}^{m-1}\left(H_{m}\right)_{j+1, j}=$ $\prod_{j=1}^{m+p-1}\left(\widetilde{H}_{m}\right)_{j+1, j}$. In accordance with Theorem 2 , the result of Theorem 3 holds for divided differences in a similar manner,

$$
t^{p}\left(\varphi_{p}\right)_{t}\left[\lambda_{1}, \ldots, \lambda_{m}\right]=\exp _{t}[\lambda_{1}, \ldots, \lambda_{m}, \underbrace{0, \ldots, 0}_{p \text { times }}] .
$$

With Theorem 2 and 3 the following equivalent formulations can be used to rewrite the scalar defect $\delta_{p, m}(t)$ defined in (3.1a).

Corollary 2 Let $\delta_{p, m}(t)$ be the scalar defect given in (3.1a) for the upper Hessenberg matrix $H_{m} \in \mathbb{C}^{m \times m}$ with positive secondary diagonal entries. Denote $0<\gamma_{m}=$ $\prod_{j=1}^{m-1}\left(H_{m}\right)_{j+1, j}$. Let $\widetilde{H}_{p, m} \in \mathbb{C}^{m+p}$ be given as in Theorem 3. For the scalar defect, we obtain the following equivalent formulations:

(i) $\delta_{p, m}(t)=\beta e_{m}^{*} t^{p} \varphi_{p}\left(t H_{m}\right) e_{1}$

(ii) $\quad=\beta \gamma_{m} t^{p}\left(\varphi_{p}\right)_{t}\left[\lambda_{1}, \ldots, \lambda_{m}\right]$

(iii) $\quad=\beta e_{m+p}^{*} \exp \left(t \widetilde{H}_{p, m}\right) e_{1}$

(iv) $\quad=\beta \gamma_{m} \exp _{t}\left[\lambda_{1}, \ldots, \lambda_{m}, 0_{p}\right]^{7}$

We remark that the eigenvalues $\lambda_{1}, \ldots, \lambda_{m}$ of the Krylov Hessenberg matrix $H_{m}$ are also referred to as Ritz values (of $A$ ) in the literature.

\section{Computable a posteriori error bounds for the Krylov propagator}

The following two propositions are used for the proof of Theorem 4 below. ${ }^{8}$

\footnotetext{
${ }^{7}$ Here, we introduce the notation $\left(\lambda_{1}, \ldots, \lambda_{m}, 0_{p}\right)=\left(\lambda_{1}, \ldots, \lambda_{m}, 0, \ldots, 0\right) \in \mathbb{C}^{m+p}$ for $p \in \mathbb{N}_{0}$.

${ }^{8}$ We use the notation introduced in the previous sections.
} 
Proposition 1 For arbitrary nodes $\lambda_{j} \in \mathbb{C}$ and $p \in \mathbb{N}_{0}$,

$$
\int_{0}^{t} s^{p}\left(\varphi_{p}\right)_{s}\left[\lambda_{1}, \ldots, \lambda_{k}\right] \mathrm{d} s=t^{p+1}\left(\varphi_{p+1}\right)_{t}\left[\lambda_{1}, \ldots, \lambda_{k}\right]
$$

Proof See Appendix B.

Proposition 2 (Lemma including (5.1.1) in [35]) For arbitrary nodes $\lambda_{j}=\xi_{j}+$ $\mathrm{i} \eta_{j} \in \mathbb{C}$,

$$
\left|\exp _{t}\left[\lambda_{1}, \ldots, \lambda_{k}\right]\right| \leq \exp _{t}\left[\xi_{1}, \ldots, \xi_{k}\right]
$$

Proof See Appendix B.

We now derive upper bounds for the error via its representation by the defect integral (3.1b).

Theorem 4 Let $p \in \mathbb{N}_{0}, \mu_{2}(A) \leq 0$, and assume that round-off errors are sufficiently small (see Corollary 1). For the eigenvalues of $H_{m}$, we write $\lambda_{j}=\xi_{j}+\mathrm{i} \eta_{j}$, $j=1, \ldots, m$. An upper bound on the error norm is given by

$$
\left\|l_{p, m}(t)\right\|_{2} \leq \beta h_{m+1, m} \gamma_{m} t\left(\varphi_{p+1}\right)_{t}\left[\xi_{1}, \ldots, \xi_{m}\right] .
$$

Proof Due to Corollary 2, (iv),

$$
\delta_{p, m}(t)=\beta \gamma_{m} \exp _{t}\left[\lambda_{1}, \ldots, \lambda_{m}, 0_{p}\right] .
$$

The divided differences in (4.2a) span over complex nodes $\lambda_{1}, \ldots, \lambda_{m}$ and $0_{p} \in \mathbb{C}^{p}$, with real parts $\xi_{1}, \ldots, \xi_{m}$. Propositions 2 and 1 imply

$\int_{0}^{t}\left|\exp _{s}\left[\lambda_{1}, \ldots, \lambda_{m}, 0_{p}\right]\right| \mathrm{d} s \leq \int_{0}^{t} \exp _{s}\left[\xi_{1}, \ldots, \xi_{m}, 0_{p}\right] \mathrm{d} s=t\left(\varphi_{1}\right)_{t}\left[\xi_{1}, \ldots, \xi_{m}, 0_{p}\right]$.

From Corollary 2, we obtain

$$
t\left(\varphi_{1}\right)_{t}\left[\xi_{1}, \ldots, \xi_{m}, 0_{p}\right]=\exp _{t}\left[\xi_{1}, \ldots, \xi_{m}, 0_{p+1}\right]=t^{p+1}\left(\varphi_{p+1}\right)_{t}\left[\xi_{1}, \ldots, \xi_{m}\right] .
$$

Equations (4.2a)-(4.2c) together with Corollary 1 imply (4.1).

For the case of $H_{m}$ having real eigenvalues, the assertion of Theorem 4 can be reformulated in the following way (see [30, Proposition 6]).

Corollary 3 Assume $\mu_{2}(A) \leq 0$ and that round-off errors are sufficiently small (see Corollary 1). For the case of $H_{m}$ having real eigenvalues $\lambda_{1}, \ldots, \lambda_{m} \in \mathbb{R}$, the upper bound on the error norm in Theorem 4 yields an exact evaluation of the defect integral. Hence,

$$
\left\|l_{p, m}(t)\right\|_{2} \leq L_{p, m}(t)=\beta h_{m+1, m} t\left(e_{m}^{*} \varphi_{p+1}\left(t H_{m}\right) e_{1}\right) .
$$


As a further corollary we formulate an upper bound on the error norm which is cheaper to evaluate compared to the bound from Theorem 4 but may be less tight. Using the Mean Value Theorem, [24, (B.26)] or [4, (44)], for the divided differences in Theorem 4 (4.1), we obtain the following result which corresponds to [30, Theorem 1 and 2]. For the exponential of a skew-Hermitian matrix, a similar error estimate has been used in [33] and is based on ideas of [44] with some lack of theory.

Corollary 4 Let $p \in \mathbb{N}_{0}, \mu_{2}(A) \leq 0$, and assume that round-off errors are sufficiently small (see Corollary 1). Let $\xi_{\max }=0$ for $p \in \mathbb{N}$ and $\xi_{\max }=\max _{j=1, \ldots, m} \xi_{j} \leq$ 0 for $p=0$ and eigenvalues $\lambda_{j}=\xi_{j}+\mathrm{i} \mu_{j} \in \mathbb{C}$ of $H_{m}$. An upper bound on the error norm is given by

$$
\left\|l_{p, m}(t)\right\|_{2} \leq \beta h_{m+1, m} \frac{\gamma_{m} t^{m} \mathrm{e}^{t \xi_{\max }}}{(m+p) !} \leq \beta h_{m+1, m} \frac{\gamma_{m} t^{m}}{(m+p) !} .
$$

For the case of $H_{m}$ having purely imaginary eigenvalues, the divided differences in Theorem 4 (see (4.1)) can be evaluated directly via [24, (B.27)],

$$
t\left(\varphi_{p+1}\right)_{t}\left[0_{m}\right]=t^{-p} \exp _{t}\left[0_{m+p+1}\right]=\frac{t^{m}}{(m+p) !} ;
$$

hence, the assertions of Theorem 4 and Corollary 4 coincide in this case.

Accuracy of the previously specified upper bounds on the error norm In the following, we again denote $\lambda_{1}, \ldots, \lambda_{m} \in \mathbb{C}$ for the eigenvalues of $H_{m}$, with $\lambda_{j}=\xi_{j}+\mathrm{i} \eta_{j}$. For the scalar defect $\delta_{p, m}(t)$ (see (3.1a)) we recapitulate Corollary 2, in particular

$$
\delta_{p, m}(t)=\beta \gamma_{m} t^{p}\left(\varphi_{p}\right)_{t}\left[\lambda_{1}, \ldots, \lambda_{m}\right]=\beta \gamma_{m} \exp _{t}\left[\lambda_{1}, \ldots, \lambda_{m}, 0_{p}\right] .
$$

Theorem 4 and its corollaries make use of the error bound given in Corollary 1 and computable upper bounds on the defect integral $L_{p, m}(t)$. A refinement of the upper bound from Corollary 1 would require further applications of the large-dimensional matrix-vector product with $A \in \mathbb{C}^{n \times n}$ and has been shown to be inefficient in terms of computational cost, see also [30, Remark 7]. The computable upper bounds on the defect integral $L_{p, m}(t)$ will be further discussed. We recapitulate the upper bound of the divided differences given in Proposition 2,

$$
\left|\exp _{t}\left[\lambda_{1}, \ldots, \lambda_{m}, 0_{p}\right]\right| \leq \exp _{t}\left[\xi_{1}, \ldots, \xi_{m}, 0_{p}\right] .
$$

Thus, in the case of $H_{m}$ having eigenvalues with a sufficiently small imaginary part, the upper bound in Proposition 2, is tight. In the following proposition, this statement is made more precise.

Proposition 3 (Part of a proof in [35], (5.2.3)) For nodes $\lambda_{j}=\xi_{j}+\mathrm{i} \eta_{j} \in \mathbb{C}$ and $t \geq 0$ with $\max _{j} t\left|\eta_{j}\right| \leq \widetilde{\eta}_{t}<\pi / 2$,

$$
0<\cos \left(\tilde{\eta}_{t}\right) \exp _{t}\left[\xi_{1}, \ldots, \xi_{k}\right] \leq\left|\exp _{t}\left[\lambda_{1}, \ldots, \lambda_{k}\right]\right| .
$$

Proof See Appendix B. 
Under the assumptions of Proposition 3, we conclude

$$
0<\cos \left(\tilde{\eta}_{t}\right) \exp _{t}\left[\xi_{1}, \ldots, \xi_{m}, 0_{p}\right] \leq\left|\exp _{t}\left[\lambda_{1}, \ldots, \lambda_{m}, 0_{p}\right]\right| .
$$

With (4.3), (4.4), (4.5), and following the proof of Theorem 4, the defect integral in $(3.1 \mathrm{~b})$ can be enclosed by

$$
\begin{aligned}
0<\cos \left(\widetilde{\eta}_{t}\right) \cdot \beta \gamma_{m} h_{m+1, m} t\left(\varphi_{p+1}\right)_{t}\left[\xi_{1}, \ldots, \xi_{m}\right] \\
\quad \leq L_{p, m}(t) \leq \beta \gamma_{m} h_{m+1, m} t\left(\varphi_{p+1}\right)_{t}\left[\xi_{1}, \ldots, \xi_{m}\right] .
\end{aligned}
$$

Hence,

$$
L_{p, m}(t)=\left(1-\mathscr{O}\left(|t \eta|^{2}\right)\right) \beta \gamma_{m} h_{m+1, m} t\left(\varphi_{p+1}\right)_{t}\left[\xi_{1}, \ldots, \xi_{m}\right],
$$

using the notation $\mathscr{O}\left(|t \eta|^{2}\right)$ in the sense of $\mathscr{O}(|t \eta|)=\mathscr{O}\left(\max _{j} t\left|\eta_{j}\right|\right)$ for $t\left|\eta_{j}\right| \rightarrow 0$. Following Proposition 3 the choice of $\widetilde{\eta}_{t}$ is independent of $\xi_{1}, \ldots, \xi_{m}$, and this carries over to the constant in (4.7).

Summarizing, we see that the defect integral can be computed exactly for the case of $H_{m}$ having real eigenvalues (Corollary 3), and a computable upper bound can be given which is tight for the case of $H_{m}$ having eigenvalues sufficiently close to the real axis (Theorem 4 and (4.7)).

The approach underlying Theorem 4 does not enable us to specify the asymptotic constant in (4.7). Therefore, we use the asymptotic expansion of the divided differences, $\left|\exp _{t}\left[\lambda_{1}, \ldots, \lambda_{m}, 0_{p}\right]\right|$ in (4.3), derived in Appendix C, to discuss the asymptotic behavior of the defect norm $\left|\delta_{p, m}(t)\right|$ for $t \rightarrow 0$. Theorem 5 from Appendix C implies

$$
\begin{aligned}
& \left|\exp _{t}\left[\lambda_{1}, \ldots, \lambda_{m}, 0_{p}\right]\right|=\frac{t^{m+p-1}}{(m+p-1) !} \exp \left(\rho_{1} t+\rho_{2} t^{2} / 2+\mathscr{O}\left(t^{3}\right)\right), \\
& \text { with } \rho_{1}=\operatorname{avg}_{p}(\xi) \text { and } \rho_{2}=\frac{\operatorname{var}_{p}(\xi)-\operatorname{var}_{p}(\eta)}{m+p+1}
\end{aligned}
$$

Here, the asymptotics holds for $t \rightarrow 0, \operatorname{avg}_{p}(\xi)=\sum_{j=1}^{m} \xi_{j} /(m+p)$ is the average, and $\operatorname{var}_{p}(\xi)=\left(\sum_{j=1}^{m}\left(\xi_{j}-\operatorname{avg}_{p}(\xi)\right)^{2}+p \operatorname{avg}_{p}(\xi)^{2}\right) /(m+p)$ is the variance of the sequence $\left\{\xi_{1}, \ldots, \xi_{m}, 0_{p}\right\}$ and $\operatorname{var}_{p}(\eta)$ is the variance of the sequence $\left\{\eta_{1}, \ldots, \eta_{m}, 0_{p}\right\}$.

Remark 6 For $H_{m}$ with purely imaginary eigenvalues $\left(\lambda_{j} \in \mathrm{i} \mathbb{R}\right)$, e.g., in the skew-Hermitian case, the following asymptotic expansion for the defect is obtained from (4.8), ${ }^{9}$

$$
\left|\delta_{p, m}(t)\right|=\beta \gamma_{m} \frac{t^{m+p-1}}{(m+p-1) !} \exp \left(-\frac{\operatorname{var}_{p}(\eta)}{2(m+p+1)} t^{2}+\mathscr{O}\left(t^{3}\right)\right) \text { for } t \rightarrow 0 .
$$

We use the expansion from (4.8) for $\left|\exp _{t}\left[\lambda_{1}, \ldots, \lambda_{m}, 0_{p}\right]\right|$ and $\exp _{t}\left[\xi_{1}, \ldots, \xi_{m}, 0_{p}\right]$ to obtain

$$
\left|\delta_{p, m}(t)\right|=\exp \left(-\frac{\operatorname{var}_{p}(\eta)}{2(m+p+1)} t^{2}+\mathscr{O}\left(t^{3}\right)\right) \cdot \beta \gamma_{m} t^{p}\left(\varphi_{p}\right)_{t}\left[\xi_{1}, \ldots, \xi_{m}\right]
$$

\footnotetext{
${ }^{9}$ It can be shown that the remainder is of even order $\mathscr{O}\left(t^{4}\right)$ in this case.
} 
Termwise integration of (4.10) and the proper prefactor gives an asymptotic expansion for the defect integral $L_{p, m}(t)$, similar to (4.7),

$L_{p, m}(t)=\left(1-\frac{\operatorname{var}_{p}(\eta)(m+p) t^{2}}{2(m+p+1)(m+p+2)}+\mathscr{O}\left(t^{3}\right)\right) \cdot \beta h_{m+1, m} \gamma_{m} t\left(\varphi_{p+1}\right)_{t}\left[\xi_{1}, \ldots, \xi_{m}\right]$

Omitting further details we state that (4.11) is to be understood in an asymptotic sense with an remainder of $\mathscr{O}\left(t^{3}|\xi||\eta|^{2}+t^{4}|\eta|^{4}\right)$. In contrast to (4.7) the remainder is depending on $\xi$ terms but (4.11) reveals further constants which can be relevant for practical applications.

Remark 7 With (4.11) we obtain a computable estimate for the relative deviation from the defect integral to the upper bound in (4.6). The criterion

$$
\operatorname{ac.est.1}(t):=\frac{\operatorname{var}_{p}(\eta)(m+p) t^{2}}{2(m+p+1)(m+p+2)}>0.1,
$$

can indicate that a tighter estimate on the defect integral could improve the error bound given in Theorem 4 in terms of accuracy. A possible choice are quadrature estimates on the defect integral, see Section 4.1 below.

A similar criterion can be given for the accuracy of the upper bound,

$$
L_{p, m}(t) \leq \beta h_{m+1, m} \gamma_{m} \frac{t^{m}}{(m+p) !},
$$

which appears in Corollary 4 (with $\xi_{\max }=0$ ) and [30, Theorem 1 and 2]. With (4.8), and $\rho_{1}$ and $\rho_{2}$ given therein, the defect integral can be written as

$L_{p, m}(t)=\beta h_{m+1, m} \gamma_{m} \frac{t^{m}}{(m+p) !}\left(1+\rho_{1} \frac{(m+p) t}{m+p+1}+\left(\rho_{1}^{2}+\rho_{2}\right) \frac{(m+p) t^{2}}{2(m+p+2)}+\mathscr{O}\left(t^{3}\right)\right)$

for $t \rightarrow 0$. In contrast to the error bound in Corollary 4 , the formulas for $\rho_{1}$ and $\rho_{2}$ in (4.8) require the evaluation of the eigenvalues of $H_{m}$. The following Proposition gives a formula for $\rho_{1}$ and $\rho_{2}$ which does not require computation of the eigenvalues of $H_{m}$ and can be evaluated on the fly.

Proposition 4 (Evaluation of $\rho_{1}$ and $\rho_{2}$ in terms of entries of $H_{m}$ ) The coefficients $\rho_{1}$ and $\rho_{2}$ in (4.8) can be rewritten as

$$
\begin{aligned}
& \rho_{1}=\frac{\operatorname{Re}\left(S_{1}\right)}{m+p}, \quad \rho_{2}=\frac{\operatorname{Im}\left(S_{1}\right)^{2}-\operatorname{Re}\left(S_{1}\right)^{2}}{(m+p)^{2}}+\frac{\operatorname{Re}\left(S_{1}^{2}+S_{2}\right)}{(m+p)(m+p+1)}, \quad \text { with } \\
& S_{1}=\sum_{j=1}^{m}\left(H_{m}\right)_{j, j} \quad \text { and } S_{2}=\sum_{j=1}^{m}\left(H_{m}\right)_{j, j}^{2}+2 \sum_{j=1}^{m-1}\left(H_{m}\right)_{j+1, j}\left(H_{m}\right)_{j, j+1} .
\end{aligned}
$$


Proof For the coefficients $\rho_{1}$ and $\rho_{2}$ we use (C.17) with $m \leftarrow m+p$ and $S_{1}$ and $S_{2}$ from (C.3). For the nodes $\lambda_{1}, \ldots, \lambda_{m}, 0_{p}$ (with $\lambda_{1}, \ldots, \lambda_{m}$ eigenvalues of $H_{m}$ ) we obtain

$$
\begin{aligned}
& S_{1}=\sum_{j=1}^{m} \lambda_{j}=\operatorname{Trace}\left(H_{m}\right)=\sum_{j=1}^{m}\left(H_{m}\right)_{j, j} \text { and } \\
& S_{2}=\sum_{j=1}^{m} \lambda_{j}^{2}=\operatorname{Trace}\left(H_{m}^{2}\right)=\sum_{j=1}^{m}\left(H_{m}\right)_{j, j}^{2}+2 \sum_{j=1}^{m-1}\left(H_{m}\right)_{j+1, j}\left(H_{m}\right)_{j, j+1} .
\end{aligned}
$$

The identity for Trace $\left(H_{m}^{2}\right)$ in (4.14) holds true due to the upper Hessenberg structure of $H_{m}$.

Following the proof of Theorem 5 we observe that the case $\rho_{1}=0$ is possible but results in $\rho_{2} \neq 0$.

Remark 8 With (4.13) and Proposition 4 we obtain a computable estimate for the relative deviation from the defect integral to the upper bound in (4.12). The criterion

$$
\text { ac.est.2( } t):=\left|\rho_{1} \frac{(m+p) t}{m+p+1}+\left(\rho_{1}^{2}+\rho_{2}\right) \frac{(m+p) t^{2}}{2(m+p+2)}\right|>0.1
$$

can indicate that a tighter estimate on the defect integral could improve the error bound given in Corollary 4 in terms of accuracy. We refer to the error bound in Theorem 4 in case the eigenvalues of $H_{m}$ have a significant real part (which can be observed via $\rho_{1}$ ).

\subsection{Quadrature-based error estimates}

First we recapitulate some prior results. In the dissipative case the integral formulation of the error from Theorem 1 can be bounded via the defect integral via Corollary 1 up to round-off. We conclude that the defect integral can be computed exactly for the case of $H_{m}$ having real eigenvalues (Corollary 3), and a computable upper bound exists which is tight for the case of $H_{m}$ having eigenvalues sufficiently close to the real axis (Theorem 4 and (4.6)).

For the case of $H_{m}$ having eigenvalues with a significant imaginary part, tight estimates are more difficult to obtain. It can be favorable to approximate the defect integral (3.1b) by quadrature to obtain an error estimate via Corollary 1 . The aim of using quadrature is to obtain an error estimate which is tighter compared to previous upper norm bounds on the error. In contrast to the proven upper error bounds given in Theorem 4, Corollary 3, and Corollary 4, the following quadrature estimates do not result in upper error bounds in general. However, in many practical cases, such quadrature estimates turn out to be still reliable.

Here, some remarks on the defect are in order to explain some subtleties with quadrature estimates for the defect integral $L_{p, m}(t)$. We discuss a test problem with a skew-Hermitian matrix $A \in \mathbb{C}^{n \times n}$. Following Remark 4 we choose $A=\mathrm{i} B$ with a 
Hermitian matrix $B$, in particularly, $B=\operatorname{tridiag}(-1,2,-1) \in \mathbb{R}^{n \times n}$ with $n=1000$. The matrix $B$ is related to a finite difference discretization of the one-dimensional negative Laplacian operator and $A$ corresponds to a free Schrödinger type problem. The eigenvalues $\sigma_{j}$, for $j=1, \ldots, n$, of $B$ are given by

$$
\sigma_{j}=4 \sin (j \pi /(2(n+1)))^{2} \text { with respective eigenvector } \psi_{j} \in \mathbb{R}^{n} .
$$

Here, $\mu_{2}(A)=0$, and the conditions of Corollary 1 hold. For a given starting vector $v \in \mathbb{C}^{n}$ the time propagation for the discretized free Schrödinger equation is given by $\exp (t A) v$ and can be approximated by the Krylov propagator with $p=0$. The following different cases for the starting vector $v$ will be discussed.

(a) Choose a random starting vector $v \in \mathbb{R}^{n}$.

(b) Start close to a linear combination of eigenvectors, $v=10^{6} \sum_{j=1}^{25} \psi_{j}+$ $\sum_{j=26}^{n} \psi_{j}$ for eigenvectors $\psi_{j}$ of the discretized negative Laplacian operator, (4.15).

(c) Start close to a linear combination of eigenvectors which are more spread on the spectrum, $v=10^{5} \sum_{j=1}^{20} \psi_{j}+10^{5} \sum_{j=n-19}^{n} \psi_{j}$ for eigenvectors $\psi_{j}$ of the discretized negative Laplacian operator, (4.15).

In addition to the setting from (a)-(c) we normalize $v,\|v\|_{2}=1$. The defect $\delta_{p, m}(t)$ for $p=0$ is computed in MATLAB, using expm to evaluate the matrix exponential of $H_{m}$ and divided differences for a fixed Krylov dimension $m=20$.

In Fig. 1 we observe $\left|\delta_{p, m}(t)\right|=\mathscr{O}\left(t^{m-1}\right.$ ) (for $t \rightarrow 0$ ) up to $t \approx 10^{1}$ for the case (a)-(c). The values of $\left|\delta_{p, m}(t)\right|$ in this time regime vary strongly among these cases. We further remark that in the case (b) for $t \geq 4 \cdot 10^{1}$ the defect $\left|\delta_{p, m}(t)\right|$ behaves similar to the divided differences of the exponential over the first eigenvalues $\lambda_{1}^{(b)}, \ldots, \lambda_{4}^{(b)}$ of $H_{m}$ with a proper prefactor. This behavior occurs if eigenvalues of $H_{m}$ are clustered, in this case $\lambda_{1}^{(b)}, \ldots, \lambda_{4}^{(b)} \approx 0$, and will be further discussed below, see Fig. 2 . For the case (c) the eigenvalues of $H_{m}$ are clustered at $\approx 0$ and $\approx 4$. Also in this case, there is a time regime for which the defect behaves similar to a lower order function in $t$ with some additional oscillations (This may be explained by the existence of different eigenvalue clusters of the same size.).

As a conclusion from the example illustrated in Fig. 1, we observe that quadrature of the defect can be relevant up to a time $t$ for which the quadrature based estimate of $\left\|l_{p, m}(t)\right\|_{2}$ (via the defect integral) is equal to a given tolerance, see (2.13). This regime of $t$ would depend on the choice of tol and additional factors such as $\beta, h_{m+1, m}$ etc. which appear in the error bound from Corollary 1. Depending on parameters and the starting vector $v$ the defect can be highly oscillatory for relevant times $t$ and, respectively, a quadrature estimate of the defect integral can be difficult to obtain. Such effects seem to be relevant for special choices of starting vectors $v$, for example case (b) and (c). The effect of $H_{m}$ having clustered eigenvalues and the prefactor used in Fig. 1 (+) are explained in the following model problem, see Fig. 2.

Divided differences with clustered nodes: an example Choose $m=3$ with nodes $a_{1}=1.123, a_{2}=1.231, a_{3}=5.43$. With this choice, we obtain cluster of nodes, $a_{1} \approx a_{2}$. For the given example, we obtain $\left|\exp _{t}\left[\mathrm{i} a_{2}, \mathrm{i} a_{3}\right]\right| \ll\left|\exp _{t}\left[\mathrm{i} a_{1}, \mathrm{i} a_{2}\right]\right|$ for $t$ 


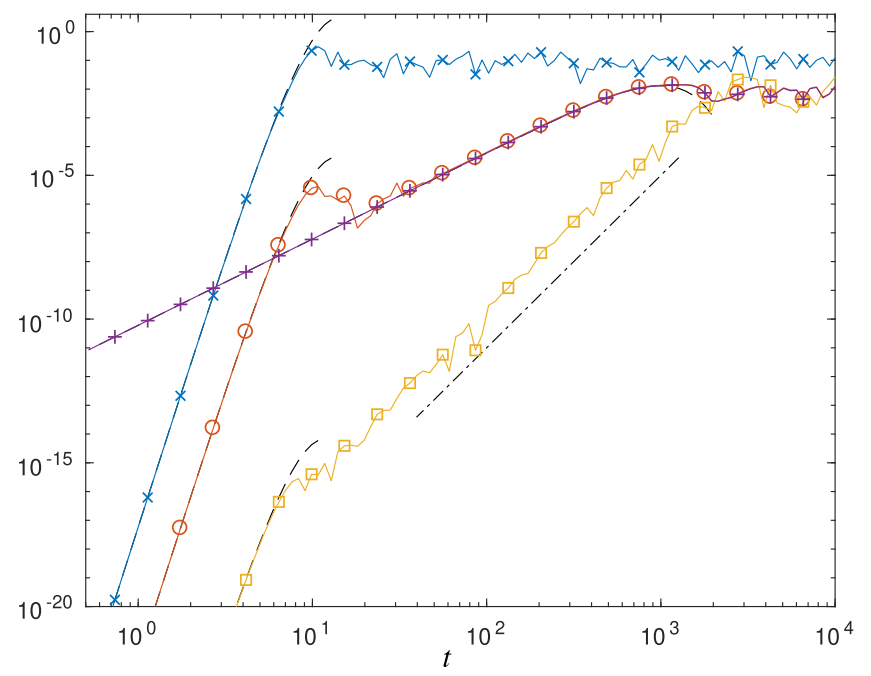

\begin{tabular}{|r|ccc|}
\hline$j$ & $\lambda_{j}^{(a)}$ & $\lambda_{j}^{(b)}$ & $\lambda_{j}^{(c)}$ \\
\hline 1 & 0.0002 & 0.0003 & 0.0001 \\
2 & 0.0422 & 0.0026 & 0.0005 \\
3 & 0.1360 & 0.0054 & 0.0013 \\
4 & 0.2712 & 0.0108 & 0.0023 \\
5 & 0.4743 & 0.3378 & 0.0032 \\
6 & 0.6921 & 0.5763 & 0.0039 \\
7 & 0.9440 & 0.8428 & 0.0054 \\
8 & 1.2105 & 1.1343 & 0.9160 \\
9 & 1.5049 & 1.4444 & 1.3768 \\
10 & 1.8318 & 1.7660 & 1.7847 \\
11 & 2.1456 & 2.0913 & 2.2385 \\
12 & 2.4621 & 2.4124 & 2.6623 \\
13 & 2.7540 & 2.7216 & 3.1348 \\
14 & 3.0393 & 3.0112 & 3.9938 \\
15 & 3.2997 & 3.2741 & 3.9961 \\
16 & 3.5088 & 3.5038 & 3.9968 \\
17 & 3.7091 & 3.6948 & 3.9977 \\
18 & 3.8402 & 3.8423 & 3.9987 \\
19 & 3.9510 & 3.9427 & 3.9995 \\
20 & 3.9945 & 3.9935 & 3.9999 \\
\hline
\end{tabular}

Fig. 1 The defect norm $\left|\delta_{p, m}(t)\right|(p=0, m=20)$ for the free Schrödinger example with different choices of starting vector case (a) ( $\times$ ), case (b) (o) and case (c) ( $\square$ ). The table on the right-hand side shows eigenvalues $\lambda_{1}^{(*)}, \ldots, \lambda_{m}^{(*)}$ of $H_{m}$ for the different starting vectors, case (a)-(c). For the case (b), the divided differences over the clustered eigenvalues $\gamma_{m}\left(\prod_{j=5}^{20} \lambda_{j}^{(b)}\right)^{-1}\left|\exp _{t}\left[\mathrm{i} \lambda_{1}^{(b)}, \ldots, \mathrm{i} \lambda_{4}^{(b)}\right]\right|$ is illustrated by (+). The asymptotic expansion of the divided differences for $t \rightarrow 0$ given in (4.9) is illustrated using dashed lines. The dash-dotted line is $\mathscr{O}\left(t^{6}\right)$

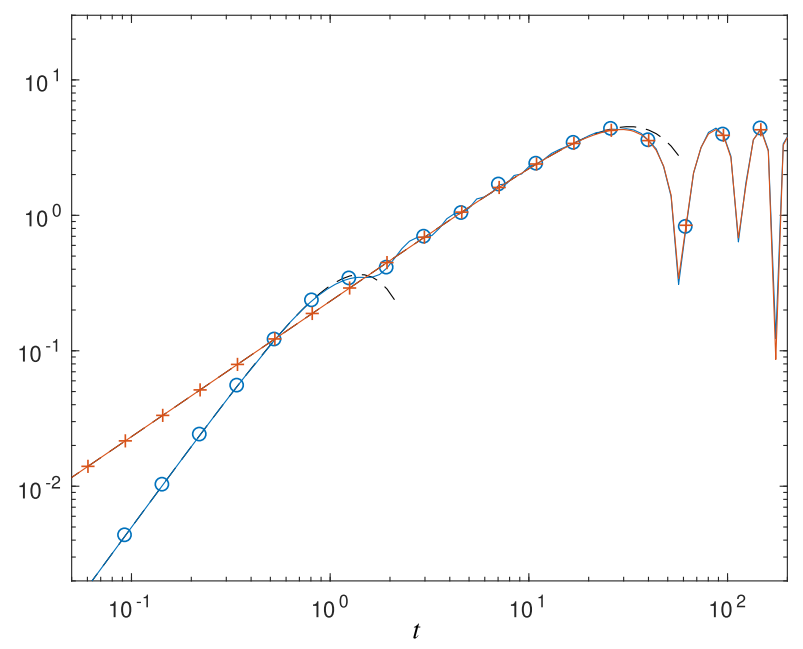

Fig. 2 The divided differences $\left|\exp _{t}\left[\mathrm{i} a_{1}, \mathrm{i} a_{2}, \mathrm{i} a_{3}\right]\right|(\mathrm{o})$ and $\left|\exp _{t}\left[\mathrm{i} a_{1}, \mathrm{i} a_{2}\right]\right| /\left|a_{3}-a_{1}\right|(+)$ for the choice of $a_{1}, a_{2}, a_{3}$ given in the text. The asymptotic expansion of the divided differences for $t \rightarrow 0$ given in (4.9) is illustrated using dashed lines 
large enough; hence, using the recursive definition of the divided differences (see [24, (B.24)] or others), we obtain

$\left|\exp _{t}\left[\mathrm{i} a_{1}, \mathrm{i} a_{2}, \mathrm{i} a_{3}\right]\right|=\left|\frac{\exp _{t}\left[\mathrm{i} a_{2}, \mathrm{i} a_{3}\right]-\exp _{t}\left[\mathrm{i} a_{1}, \mathrm{i} a_{2}\right]}{a_{3}-a_{1}}\right| \approx\left|\frac{\exp _{t}\left[\mathrm{i} a_{1}, \mathrm{i} a_{2}\right]}{a_{3}-a_{1}}\right|$, for larger $t$.

This example is illustrated in Fig. 2. This behavior can be generalized for a larger number of nodes and is also observed in Fig. 1.

Quadrature estimates for the defect integral With the previous observations on the defect we now discuss different quadrature-based estimates.

The generalized residual estimate, which was introduced in [28] and appeared in a similar manner in $[5,11,34,46]$, conforms to a quadrature on the defect norm integral which is related to the error norm via Corollary 1.

Remark 9 (Generalized residual estimate, see also [28]) Applying the right-endpoint rectangle rule we have

$$
\int_{0}^{t}\left|\delta_{p, m}(s)\right| \mathrm{d} s \approx t\left|\delta_{p, m}(t)\right|,
$$

and with Corollary 1 (and $\delta_{p, m}(t)$ given in (3.1a)) we obtain the error estimate

$$
\left\|l_{p, m}(t)\right\|_{2} \approx h_{m+1, m} t^{1-p}\left|\delta_{p, m}(t)\right|=\beta h_{m+1, m} t\left|e_{m}^{*} \varphi_{p}\left(t H_{m}\right) e_{1}\right| .
$$

Assume that $\max _{s \in[0, t]}\left|\delta_{p, m}(s)\right|=\left|\delta_{p, m}(t)\right|$, e.g., $\left|\delta_{p, m}(t)\right|$ is monotonically increasing in $t$. Then,

$$
\int_{0}^{t}\left|\delta_{p, m}(s)\right| \mathrm{d} s \leq t \max _{s \in[0, t]}\left|\delta_{p, m}(s)\right|=t\left|\delta_{p, m}(t)\right| .
$$

In this case, the generalized residual estimate from Remark 9 results in an upper bound on the error norm.

In the most general case, the defect is of high order for $t \rightarrow 0$ and in a relevant time regime, see also Fig. 1 case (a) and previous remarks. Then, the defect is a higher order function and the right-endpoint quadrature does result in an upper bound but is not tight. In this case, we can improve the estimate by a prefactor depending on the effective order defined in Appendix C. If the defect is sufficiently smooth in a relevant time regime, this results in a tight upper bound on the error norm.

Remark 10 (Effective order estimate, see also [30]) Denote $f(t)=$ $\left|\exp _{t}\left[\lambda_{1}, \ldots, \lambda_{m}, 0_{p}\right]\right|$ for the time-dependent part of the defect with eigenvalues $\lambda_{1}, \ldots, \lambda_{m}$ of $H_{m}$. Assume $f(t)>0$ for a sufficiently small time regime $t>0$. We consider the effective order $\rho(t)$ defined in (C.4a). With the following estimate for the integral of the defect,

$$
\int_{0}^{t}\left|\delta_{p, m}(s)\right| \mathrm{d} s \approx \frac{t}{\rho(t)+1}\left|\delta_{p, m}(t)\right|
$$


and from Corollary 1 (with $\delta_{p, m}(t)$ given in (3.1a)), we obtain

$$
\left\|l_{p, m}(t)\right\|_{2} \approx h_{m+1, m} \frac{t^{1-p}}{\rho(t)+1}\left|\delta_{p, m}(t)\right|=\beta h_{m+1, m} \frac{t}{\rho(t)+1}\left|e_{m}^{*} \varphi_{p}\left(t H_{m}\right) e_{1}\right| .
$$

In [30], the effective order is defined for $\left|e_{m}^{*} \mathrm{e}^{t H_{m}} e_{1}\right|(p=0)$ which is equivalent to the definition via the divided differences of $f(t)$. (This follows from Corollary 2 and the definition of the effective order which is independent of a constant prefactor.)

Some of the following observations already appeared in [30]. The quadrature scheme in Remark 10 is motivated by the following relation of the effective order and the integral of the divided differences $f(t)$. From (C.4a),

$$
f(t)=\frac{f^{\prime}(t) t}{\rho(t)} .
$$

Integration and application of the mean value theorem shows the existence of $t^{*} \in$ $[0, t]$ with

$$
\int_{0}^{t} f(s) \mathrm{d} s=\frac{1}{\rho\left(t^{*}\right)} \int_{0}^{t} f^{\prime}(s) s \mathrm{~d} s,
$$

and integration by parts gives

$$
\int_{0}^{t} f(s) \mathrm{d} s=\frac{t f(t)}{1+\rho\left(t^{*}\right)} .
$$

This result can passed over to the integral of the defect.

Assume the effective order is monotonically decreasing for $t$ small enough, with $\min _{s \in(0, t]} \rho(s)=\rho(t) \geq 0$. This holds in an asymptotic regime for the dissipative case up to round-off, see also Theorem 5 with the real parts $\xi_{1}, \ldots, \xi_{m}$ of the eigenvalues of $H_{m}$ being non-positive. With (4.16) and the assumption $0 \leq \rho(t) \leq \rho(s) \leq$ $m+p-1=\rho(0+)$ for $s \in[0, t]$, we inclose the integral of the defect by

$$
\frac{t}{m}\left|\delta_{p, m}(t)\right| \leq \int_{0}^{t}\left|\delta_{p, m}(s)\right| \mathrm{d} s \leq \frac{t}{\rho(t)+1}\left|\delta_{p, m}(t)\right| \leq t\left|\delta_{p, m}(t)\right| .
$$

Combining (4.17) and Corollary 1, we obtain the upper bound

$$
\left\|l_{p, m}(t)\right\|_{2} \leq \frac{h_{m+1, m} t^{1-p}}{\rho(t)+1} \cdot\left|\delta_{p, m}(t)\right| \leq h_{m+1, m} t^{1-p} \cdot\left|\delta_{p, m}(t)\right| .
$$

A computable expression for the effective order was given in $[30,(6.10)]$. This result can be generalized to the case $p \in \mathbb{N}_{0}$,

$\rho(t)=\left\{\begin{array}{ll}t \operatorname{Re}\left(\left(H_{m}\right)_{m, m}+\left(H_{m}\right)_{m, m-1}\left(y_{p, m}(t)\right)_{m-1} /\left(y_{p, m}(t)\right)_{m}\right) & \text { for } p=0, \\ \operatorname{Re}\left(\left(y_{p-1, m}(t)\right)_{m} /\left(y_{p, m}(t)\right)_{m}\right) & \text { for } p \in \mathbb{N},\end{array}\right.$ and

with $y_{p, m}(t) \in \mathbb{C}^{m}$ from (2.9). The expression for the case $p \in \mathbb{N}$ can be obtained by $[30,(6.10)]$ applied on the representation $\left|e_{m+p}^{*} \mathrm{e}^{t \widetilde{H}_{m}} e_{1}\right|$ for the defect ((iii). in Corollary 2) and making use of the special structure of $\widetilde{H}_{m}, \beta e_{m+p}^{*} \mathrm{e}^{t \widetilde{H}_{m}} e_{1}=t^{p}\left(y_{p, m}(t)\right)_{m}$ (see Corollary 2) and $\beta e_{m+p-1}^{*} \mathrm{e}^{t \widetilde{H}_{m}} e_{1}=$ $t^{p-1}\left(y_{p-1, m}(t)\right)_{m}$ (see [49, Corollary 1]). 
As illustrated in Fig. 1 the defect can be highly oscillatory in a relevant time regime, especially for specific starting vectors, and in this case the quadrature estimates should be handled with care.

\subsection{A stopping criterion for the lucky breakdown}

The special case $h_{k+1, k}=0$ during the construction of the Krylov subspace is considered to be a lucky breakdown, a breakdown of the Arnoldi or Lanczos iteration with the benefit of an exact approximation of $\varphi_{p}(t A) v$ for any $t>0$ via the Krylov subspace $\mathscr{K}_{k}(A, v)$. In floating point arithmetic, the lucky breakdown results in $h_{k+1, k} \approx 0$ and can lead to stability issues if the Arnoldi or Lanczos method is not stopped properly. The condition that the Krylov propagator is exact is not exactly determinable in floating point arithmetic but can be weakened to the error condition in (2.13) for a given tolerance tol per unit step. With this approach, we introduce a stopping criterion which can be applied on the fly to detect a lucky breakdown and satisfies an error bound. This does not depend on any a priori information as long the tolerance tol is chosen properly so that round-off errors can be neglected, see remarks before Corollary 1.

Proposition 5 Let $\mu_{2}(A) \leq 0$ and assume that round-off errors are sufficiently small, see Corollary 1. Let tol be a given tolerance and

$$
\frac{\beta h_{k+1, k}}{(p+1) !} \leq \text { tol }
$$

be satisfied at the kth step of the Arnoldi or Lanczos iteration. Then, the iteration can be stopped and the Krylov subspace $\mathscr{K}_{k}(A, v)$ can be used to approximate the vector $\varphi_{p}(t A) v$ with a respective error per unit step $\left\|l_{p, k}(t)\right\|_{2} \leq t \cdot$ tol.

Proof We use the upper bound on the error norm from Corollary 1,

$$
\left\|l_{p, k}(t)\right\|_{2} \leq \frac{h_{k+1, k}}{t^{p}} \int_{0}^{t}\left|\delta_{p, k}(s)\right| \mathrm{d} s .
$$

To obtain a uniform bound on the defect integral, we use

$$
\left|\delta_{p, k}(t)\right| \leq \beta t^{p}\left\|e_{k}\right\|_{2}\left\|\varphi_{p}\left(t H_{k}\right) e_{1}\right\|_{2}=\beta t^{p}\left\|\varphi_{p}\left(t H_{k}\right) e_{1}\right\|_{2} .
$$

- For $p>0$, we apply the integral representation (2.3) on $\varphi_{p}\left(t H_{m}\right) e_{1}$ to obtain the upper bound

$$
\left\|\varphi_{p}\left(t H_{m}\right) e_{1}\right\|_{2} \leq \frac{\max _{s \in[0, t]}\left\|\mathrm{e}^{s H_{m}}\right\|_{2}}{(p-1) !} \int_{0}^{1} \theta^{p-1} \mathrm{~d} \theta=\frac{\max _{s \in[0, t]}\left\|\mathrm{e}^{s H_{m}}\right\|_{2}}{p !} .
$$

- For $p=0$, the analogous result is directly obtained: Combine (4.20) and (4.21) with $\left\|\mathrm{e}^{s H_{k}}\right\|_{2} \leq \mathrm{e}^{t \mu_{2}\left(H_{k}\right)} \leq \mathrm{e}^{t \mu_{2}(A)}$ up to round-off and $\mu_{2}(A) \leq 0$, giving

$$
\left|\delta_{p, k}(t)\right| \leq \beta \frac{t^{p}}{p !}, \quad \text { and } \int_{0}^{t}\left|\delta_{p, k}(s)\right| \mathrm{d} s \leq \beta \frac{t^{p+1}}{(p+1) !} .
$$


Together with (4.19) and (4.18), we conclude $\left\|l_{p, k}(t)\right\|_{2} \leq t \cdot$ tol.

\section{Numerical experiments}

The notation for the error $l_{p, m}(t)$, the estimate of the error norm $\zeta_{p, m}(t)$ and the tolerance tol have been introduced in (2.12) and (2.13). The notation $\zeta_{p, m}$ will be used for different choices of error estimates discussed in the previous section. Theorem 4 and Corollary 4 result in upper bounds on the error norm, $\left\|l_{p, m}(t)\right\|_{2} \leq \zeta_{p, m}(t)$. The quadrature-based error estimates given in Remark 9 and 10 result in estimates for the error norm, $\left\|l_{p, m}(t)\right\|_{2} \approx \zeta_{p, m}(t)$, and with additional conditions also give upper bounds.

For a fixed tolerance tol, we use the notation $t(m)$ for the smallest time $t$ with $\zeta_{p, m}(t)=t \cdot$ tol, see (2.13). This choice of $t(m)$ helps us to verify the tested error estimates for a time $t$ which is of the most practical interest. With the help of a reference solution, the true error norm per unit step can be tested by $\left\|l_{p, m}(t(m))\right\|_{2} / t(m)$.

We also consider the following previously known error estimates in our numerical experiments. The generalized residual estimate [28] was recapitulated in Remark 9 and will be discussed in the numerical experiments. Furthermore, we test the performance of the error bound given in [10, Proposition 6]. This upper bound on the error norm applies to the Krylov approximation of $\varphi_{p}(-t A) v$ for $p \in \mathbb{N}_{0}$, a matrix $A \in \mathbb{R}^{n \times n}$ with a numerical range in the right complex half-plane (up to a potential shift), and $v \in \mathbb{R}^{n}$. In this case, the matrix $A$ can have real and complex eigenvalues, where the latter come in complex conjugate pairs. Concerning the skew-Hermitian case, a similar error bound for the Krylov approximation to $\varphi_{p}(-\mathrm{i} t B) v$ for a Hermitian matrix $B \in \mathbb{R}^{n \times n}$ and $p \in \mathbb{N}_{0}$ is given separately in [10, Proposition 8]. To evaluate these error bounds the eigenvalues of $H_{m}$ and the terms $h_{m+1, m}$ and $\gamma_{m}$ are used.

A series expansion for the error concerning $\varphi$-functions is given in [49, Theorem 2] and the leading terms of this expansion can be used for error estimation, cf. [41, 49]. In general [49] suggests to evaluate more than one term of this series to ensure reliability of the obtained error estimate, which requires further matrix-vector multiplications in the given large dimensional space. This can often be inefficient in terms of computational cost, cf. [30, Remark 7], and we avoid this series expansion in the general case. However, when the Ritz values are realvalued, the error bound in Corollary 3 (corresponding to the bound in Theorem 4) coincides with the leading term of the error series in [49, Theorem 2]. Thus, the first term of the error series in [49, Theorem 2] yields a reliable error bound in this case. For the convection-diffusion equation with parameter $v=100$ in Section 5.1 below (the Ritz values have negligible imaginary parts in this case), the error bound of Theorem 4 performs well (comparable to the effective order estimate and better than the other error estimates considered, e.g., the generalized residual estimate), and this potentially carries over to the error estimates in $[41,49]$. 


\subsection{Convection-diffusion equation}

Consider the following two-dimensional convection-diffusion equation with $t \geq 0$ and $x \in[0,1]^{2}$,

$$
\partial_{t} u=L u, \quad \text { with } L=\Delta+v\left(\partial_{x_{1}}+\partial_{x_{2}}\right), \quad u=u(t, x), v \in \mathbb{R} .
$$

Let $A \in \mathbb{R}^{n \times n}$ be obtained by the two-dimensional finite difference discretization of the operator $L$ in (5.1) with zero Dirichlet boundary conditions and $N=500$ inner mesh points in each spatial direction, hence, $n=N^{2}$. This test problem is similar to other convection-diffusion equations appearing in the study of Krylov subspace methods, see also [6, 15, 19, 30] and others.

For the convection parameter we choose $v=100,500$ which results in a nonnormal matrix $A$. Considering the spectrum of $A$ the case $v=100$ is closer to the Hermitian case and $v=500$ is closer to the skew-Hermitian case. In both cases, the numerical range of $A$ is contained in the left complex plane, $\mu_{2}(A) \leq 0$.

We discuss error estimates for the Krylov approximation of the matrix exponential $(p=0)$ and a $\varphi$-function (for which we choose $p=2$ ). For the case $p=0$, the action of the matrix exponential $\mathrm{e}^{t A} v$ is approximated in the Krylov subspace $\mathscr{K}_{m}(A, v)$, see (2.8b). Analogously, for the case $p=2$ we approximate $\varphi_{p}(t A) v$ as given in (2.8a). As a starting vector we choose the normalized vector $v=(1 / N, \ldots, 1 / N)^{*} \in \mathbb{R}^{n}$. In Fig. 3, we compare the error bounds given in Theorem 4, Corollary 4 and [10, Proposition 6], and the generalized residual estimate (Remark 9) and the effective order estimate (Remark 10), for the convectiondiffusion equation. The error bound of Corollary 4 is applied with $\xi_{\max }=0$ (the effect of $\xi_{\max }$ is negligible for the current examples). Concerning the error bound given in [10, Proposition 8], we choose the parameter $\varepsilon$ by minimizing [10, (39)], and $a=0$.

For the case $v=100$ the eigenvalues of $H_{m}$ have a negligible imaginary part and the upper bound given in Theorem 4 constitutes a tight upper bound on the exact evaluation of the scaled defect integral, which yields a tight error bound. This error bound and the effective order estimate (Remark 10), which is based on a quadrature estimate on the defect integral, yield approximately the same results for the case $v=100$. The performance of the generalized residual estimate (Remark 9) is similar to the performance of the error bound in [10, Proposition 6], especially for larger choices of $m$. The error bound in Corollary 4 is only accurate for small $m$ in the current example. The high accuracy of the error bound in Theorem 4 and the effective order estimates results in time steps $t(m)$ which are larger than the time steps suggested by generalized residual estimate and the error bound in [10, Proposition 6], and significantly larger compared to the time steps given by Corollary 4. Comparing the cases $p=0$ and $p=2$, the time steps suggested by the error bounds of Corollary 4 and [10, Proposition 6] are slightly smaller in relation to the time step prescribed by the effective order estimate for $p=2$. Considering the true error for the time steps computed by the error bound in Theorem 4, the effective order estimate and the generalized residual estimate, the performance of these estimates only differs slightly between the cases $p=0$ and $p=2$. 

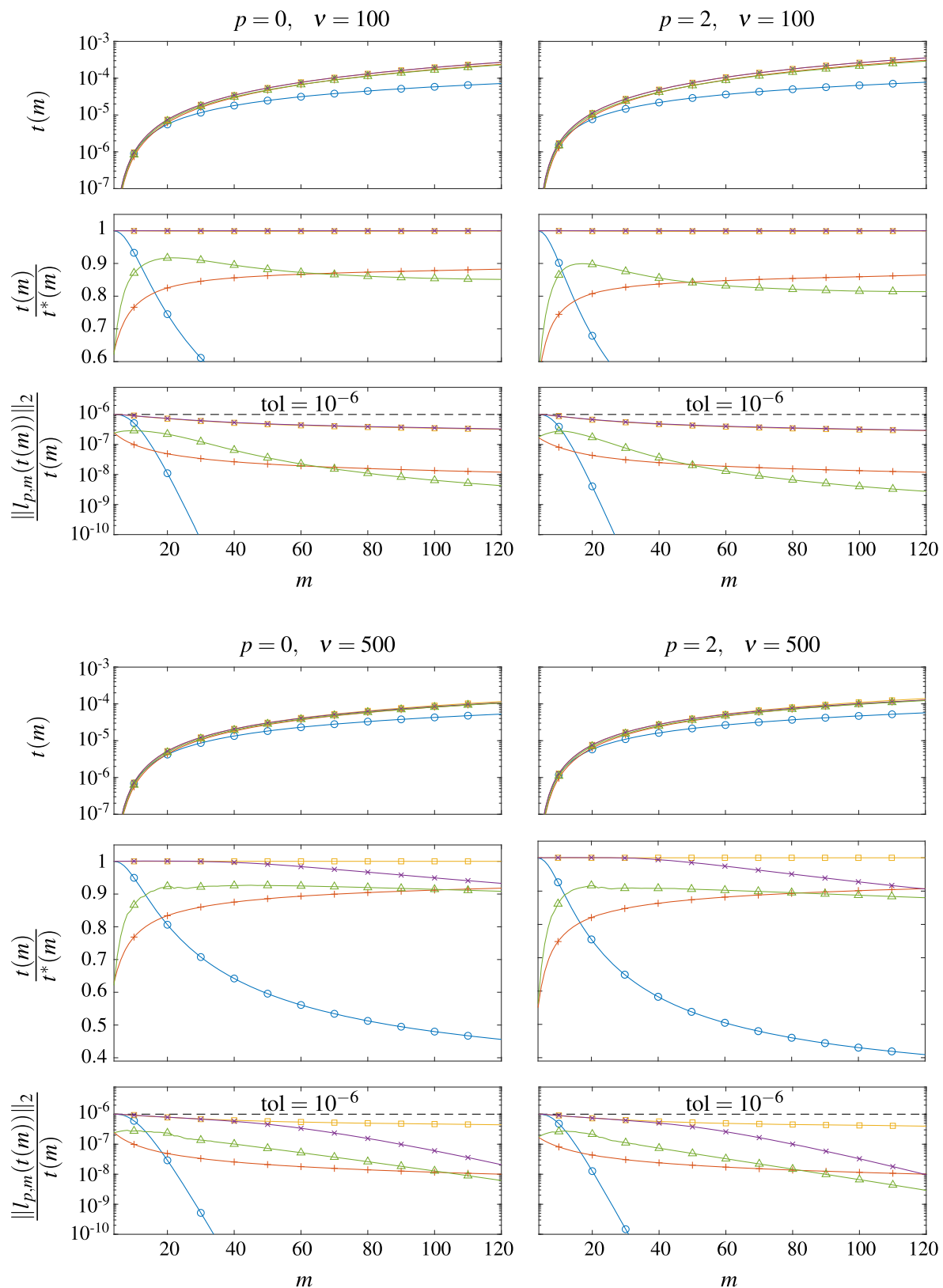

Fig. 3 Convection-diffusion problem (5.1) for the parameter $v=100$ (top) and $v=500$ (bottom). For each choice of $v$ we consider $p=0$ (left) and $p=2$ (right). Three rows of plots are addressed to each choice of $v$ : The first row shows the time $t(m)$ which is the smallest $t$ such that $\zeta_{p, m}(t)=t \cdot$ tol for tol $=10^{-6}$ and $\zeta_{p, m}$ corresponding to the error bound given in Theorem $4(\times)$, Corollary $4(\circ)$, the generalized residual estimate given in Remark $9(+)$, the effective order estimate given in Remark 10 $(\square)$, and the error bound given in [10, Proposition 6] $(\triangle)$. For the second row we choose $t^{*}(m)$ as the largest time step $t(m)$ given by the currently discussed error estimate, and we show $t(m) / t^{*}(m)$ for $t(m)$ as chosen above. The third row shows the true error per unit step, $\left\|l_{p, m}(t(m))\right\|_{2} / t(m)$, for the time $t(m)$ as chosen above 
For the case $v=500$, the matrix $H_{m}$ has eigenvalues with larger imaginary parts (especially for larger $m$ ). In this case the error bound in Theorem 4, is less tight, and the effective order estimate (Remark 10) performs best comparing to the other error estimates. Comparing the cases $p=0$ and $p=2$, we observe that the time steps suggested by the error bounds of Theorem 4, Corollary 4 and [10, Proposition 6] are slightly smaller in relation to the time step of the effective order estimate for $p=2$.

The criterion ac.est.1 $(t)$ given in Remark 7 is evaluated for $v=100,500$ and $p=0,2$ with $t(m)$ corresponding to Theorem 4 (see caption of Fig. 3). For $v=100$ we obtain ac.est. $1(t(m))<0.1$ for any $m$ tested and $p=0,2$. For $v=500$ the smallest $m$ with ac.est.1 $(t(m))>0.1$ is $m=40$ and $m=36$ for $p=0$ and $p=2$, respectively. The error bound in Theorem 4 conforms to an upper bound of the scaled defect integral, and in the case of ac.est.1 $(t(m))>0.1$ a more accurate estimate on the defect integral is likely to perform better. For $v=500$ and $m=40(p=0)$ and $m=36(p=2)$, we observe that this is the case for the effective order estimate. Similar to the criterion ac.est.1 $(t)$, we test ac.est.2( $t)$ given in Remark 8 for $t(m)$ according to Corollary 4. For $v=100$ the smallest $m$ with ac.est. $2(t(m))>0.1$ is $m=7$ for $p=0,2$ individually. Otherwise, for $v=500$ the smallest $m$ with ac.est. $2(t(m))>0.1$ is $m=8$ and $m=7$ for $p=0$ and $p=2$, respectively.

\subsection{Free Schrödinger equation, a skew-Hermitian problem}

For the free Schrödinger equation, we let $A$ be a finite difference discretization of the Laplace operator, precisely, we choose $A$ corresponding to $L$ in (5.1) with $v=0$ and $N=500$. With $A$ corresponding to a discretized Laplace operator, the vector $\mathrm{e}^{\mathrm{i} t A} v$ yields a solution to a discretized free Schrödinger equation with starting vector $v$. The free Schrödinger equation represents a skew-Hermitian problem, and following Remark 4 we approximate $\mathrm{e}^{\mathrm{i} t A} v$ in the Krylov subspace $\mathscr{K}_{m}(A, v)$ by $\beta V_{m} \mathrm{e}^{\mathrm{i} t H_{m}} e_{1}$. Analogously to the previous subsection, we choose the normalized starting vector $v=(1 / N, \ldots, 1 / N)^{*} \in \mathbb{R}^{n}$, and we also consider the Krylov approximation to $\varphi_{p}(\mathrm{i} t A) v$ for $p=2$, i.e., $\beta V_{m} \varphi_{p}\left(\mathrm{i} t H_{m}\right) e_{1}$.

In Fig. 4, the error bounds given in Corollary 4 (which coincides with the error bound given in Theorem 4 in the skew-Hermitian case) and [10, Proposition 8] (the counterpart to [10, Proposition 6] for the skew-Hermitian case), the effective order estimate (Remark 10), and the generalized residual estimate (Remark 9) are evaluated for the current example. For the parameter $\varepsilon$ in [10, Proposition 8], we choose $\varepsilon=$ $m / t$ as suggested in the numerical experiments therein.

For the skew-Hermitian case, the effective order estimate (Remark 10) yields the largest time steps compared to the other error estimates. The error bound of Corollary 4 performs well for moderate $m$ and better than the error bound in [10, Proposition 8] for any of the tested $m$ here. For larger $m$ the generalized residual estimate performs better than the error bound of Corollary 4. Similar to examples of the previous subsection, the error bound of Corollary 4 performs better for the case $p=0$ compared to $p=2$. Similar results can be observed for the error bound of [10, Proposition 8]. The performance of the effective order estimate and the generalized residual estimate only differs slightly between the cases $p=0$ and $p=2$. 

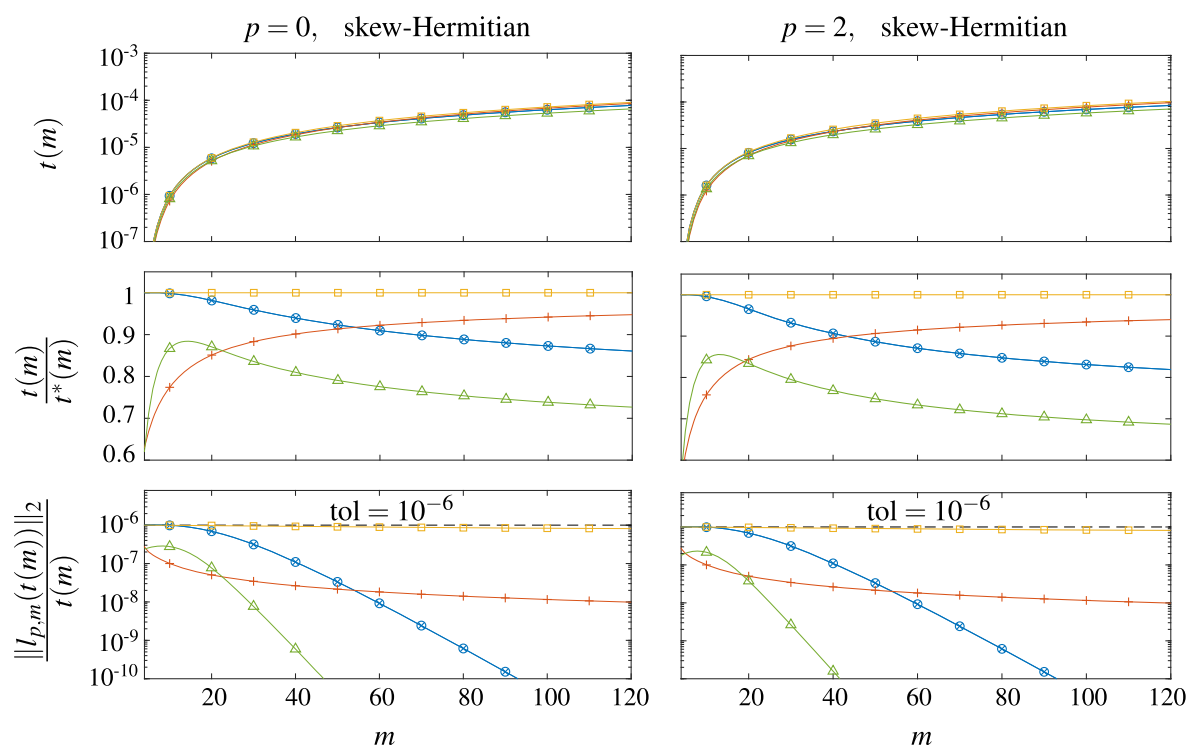

Fig. 4 The skew-Hermitian problem $\varphi_{p}$ (i $\left.A\right) v$ where $A$ corresponds to the Laplace operator $((5.1)$ with $v=0)$ and $v=(1 / N, \ldots, 1 / N)^{*}$. Results are shown for $p=0$ (left) and $p=2$ (right). For $p=$ 0 this problem is related to the free Schrödinger equation. The top row shows the time $t(m)$ which is the smallest $t$ such that $\zeta_{p, m}(t)=t \cdot$ tol for tol $=10^{-6}$ and $\zeta_{p, m}$ corresponding to the error bound given in Theorem $4(\times)$, Corollary $4(\circ)$, the generalized residual estimate given in Remark $9(+)$, the effective order estimate given in Remark $10(\square)$, and the error bound given in [10, Proposition 8] $(\triangle)$. The error bounds in Theorem $4(\times)$ and Corollary $4(\circ)$ coincide in the skew-Hermitian case. For the middle row we choose $t^{*}(m)$ as the largest time step $t(m)$ given by the currently discussed error estimate, and we show $t(m) / t^{*}(m)$ for $t(m)$ as chosen above. The bottom row shows the true error per unit step, $\left\|l_{p, m}(t(m))\right\|_{2} / t(m)$, for the time $t(m)$ as chosen above

We test ac.est.2(t) given in Remark 8 for $t(m)$ according to Corollary 4 . The smallest $m$ with ac.est.2( $t(m))>0.1$ is $m=15$ and $m=13$ for $p=0$ and $p=2$, respectively. Following Remark 8, the error bound given in Corollary 4 overestimates the error by a factor 1.1 (in an asymptotic sense) for these values of $m$, which fits to the results shown in Fig. 4.

\subsection{Free Schrödinger equation with a double well potential and a Gaussian wave packet as an initial state}

In the following numerical experiment, we choose a special starting vector which results in the matrix $H_{m}$ having clustered eigenvalues, and we observe effects which were previously discussed in Section 4.1. Typically, this is related to regularity properties of the underlying initial state.

We consider the one-dimensional free Schrödinger equation with a double well potential,

$\partial_{t} \psi=-\mathrm{i} H \psi, \quad$ with $H=-\Delta+V, \quad \psi=\psi(t, x) \in \mathbb{C}, V=V(x) \in \mathbb{R}$, 
for $t \geq 0, x \in[-10,10]$ and $V(x)=x^{4}-15 x^{2}$. Let $B \in \mathbb{C}^{n \times n}$ be the discretized version of the Hamiltonian operator $H$ in (5.2) with periodic boundary conditions using a finite difference scheme with a mesh of size $n=10000$. With $B$ Hermitian, the full problem $A=-\mathrm{i} B$ is skew-Hermitian (see Remark 4) with $\mu_{2}(A)=0$. For the initial state of (5.2) we choose a Gaussian wave packet,

$$
\psi(t=0, x)=(0.2 \pi)^{-1 / 4} \exp \left(-(x+2.5)^{2} /(0.4)\right),
$$

which is evaluated on the mesh and normalized to obtain a discrete starting vector $v \in \mathbb{R}^{n}$. This problem also appears in $[29,51]$.

We discuss error estimates for the case $p=0$ (Krylov approximation of $\mathrm{e}^{-\mathrm{i} t B} v$ ). The implementation of the skew-Hermitian problem is described in Remark 4. In Fig. 5 the upper bound given in Corollary 4 (which coincides with the error bound given in Theorem 4 for the skew-Hermitian case) and the error estimates given in
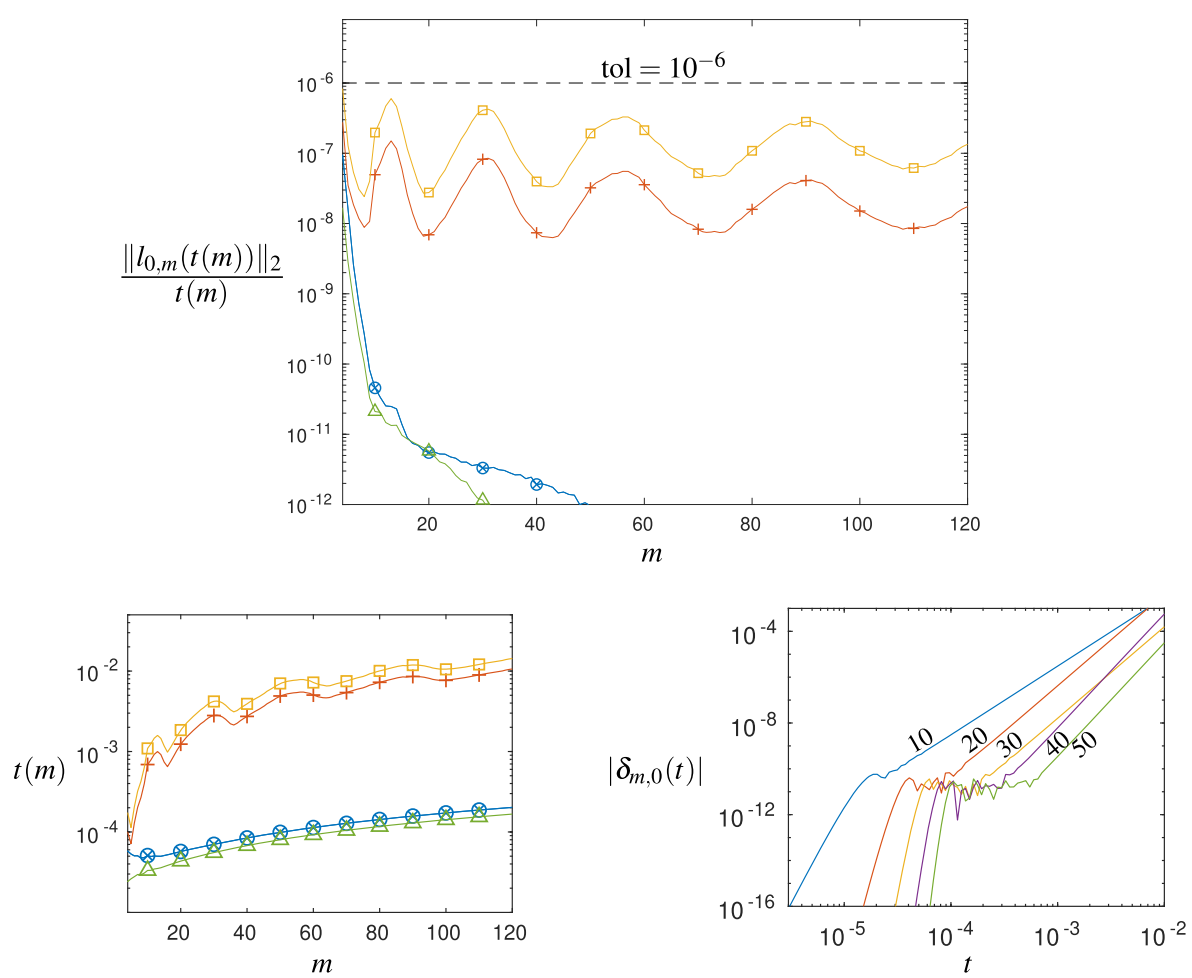

Fig. 5 Results for the free Schrödinger problem with a double well potential and the starting vector given by (5.3). This figure shows the time $t(m)$ (bottom left), which is the smallest $t$ so that $z$ et $a_{0, m}(t)=t \cdot t o l$ for $t o l=10^{-6}$, the true error per unit step (top) $\left|l_{0, m}(t(m))\right|_{2} / t(m)$ and the defect norm $\mid$ delta $_{m, 0}(t) \mid$ (bottom right) for $\min 10,20,30,40,50$. The results for $t(m)$ and $\left\|l_{0, m}(t(m))\right\|_{2} / t(m)$ are given for $\zeta_{0, m}$ being the upper norm bound given in Theorem $4(\times)$, Corollary $4(0)$, the generalized residual estimate given in Remark $9(+)$, the effective order estimate given in Remark 10 ( $\square$ ) and the error bound given in [10, Proposition 8] $(\triangle)$. The results for Theorem $4(\times)$ and Corollary 4 (०) coincidence in the skewHermitian case 
Remark 9 and 10 are compared. Additionally, we consider the error bound given in [10, Proposition 8] with the parameter choice $\varepsilon=m / t$.

The error bounds given in Corollary 4 and [10, Proposition 8] are reliable but not tight for the current example. Thus, the time steps $t(m)$ which are suggested by these error bounds are significantly smaller than the time steps suggested by the quadrature-based error estimates (Remarks 9 and 10), and comparing with the numerical experiments of the previous subsection, this seems to be highly affected by the choice of the starting vector. For the error bound in Corollary 4, this can be explained by the loss of order of the defect. However, the error bound in Corollary 4 shows a better performance compared to the error bound in [10, Proposition 8].

In terms of accuracy, the effective order estimate (Remark 10) performs significantly better compared to the error bounds in Corollary 4 and [10, Proposition 8], and better compared to the generalized residual estimate (Remark 9). In terms of reliability, we have argued that the effective order estimate and the generalized residual estimate constitute upper bounds on the error norm when the defect norm behaves sufficiently smooth. The defect norm $\left|\delta_{m, 0}(t)\right|$, which is presented in the lower right corner of Fig. 5, does have an oscillatory behavior in a specific time regime which can be related to the starting vector, cf. Section 4.1. For the time steps which are relevant for the current example, this does not critically affect the quadrature estimates on the defect integral related to Remark 9 and 10. Under certain conditions, e.g., a different choice for the tolerance tol, this oscillatory behavior of the defect can lead to failure of the error estimates given in Remark 9 and 10. However, the quadrature of the defect integral can be further improved in such cases to ensure a reliable error estimate.

\section{Conclusions and outlook}

In this work, various a posteriori bounds and estimates on the error norm, which have their origin in an integral representation of the error using the defect (residual), are studied. We have characterized the accuracy of these error bounds by the positioning of Ritz values (i.e., eigenvalues of $H_{m}$ ) on the complex plane. The case of real Ritz values is the most favorable one to obtain a tight error bound via an integral on the defect norm (Corollary 3). A new error bound (Theorem 4) has shown to be tight if Ritz values are close to the real axis and in this case favorably compares with existing error bounds. We further recapitulate an existing error bound (Corollary 4) which remains relevant, especially for the case of Ritz values with a significant imaginary part. In addition for the error bound in Theorem 4 and Corollary 4, we have provided a criterion to quantify the achieved accuracy on the fly. For an illustration of the claims concerning the new error bound, we primary refer to the numerical example given in Section 5.1. The quadrature-based error estimates in Section 4.1 (e.g., the generalized residual estimate) do not yield proven upper bounds on the error norm and we addressed special cases (e.g., the numerical example in Section 5.3) for which the reliability of these estimates can be problematic. These cases are also analyzed in terms of Ritz values in Section 4.1 and this relation can be of further interest 
for a numerical implementation. Nevertheless, in most cases, the quadrature-based estimates remain valid, whereat the effective order quadrature stands out in terms of performance.

We also remark that the theory provided in our work gives the possibility to adapt the choice of the error estimate on the fly to obtain an estimate which is as reliable, accurate and economic as possible. This is the topic of further work.

\section{Appendix A: Properties of the Krylov subspace in exact and floating point arithmetic}

Let $H_{m}=V_{m}^{*} A V_{m}$ and $V_{m}^{*} V_{m}=I_{m \times m}$ in exact arithmetic. For $z \in \mathrm{W}\left(H_{m}\right)$ (numerical range of $H_{m}$ ), there exists $x \in \mathbb{C}^{m}$ with

$$
z=\frac{x^{*} H_{m} x}{x^{*} x}=\frac{x^{*} V_{m}^{*} A V_{m} x}{x^{*} V_{m}^{*} V_{m} x}=\frac{y^{*} A y}{y^{*} y}, \text { for } y=V_{m} x,
$$

whence $\mathrm{W}\left(H_{m}\right) \subseteq \mathrm{W}(A)$.

Similar results hold in floating point arithmetic with relative machine precision $\varepsilon$ and certain additional assumptions. Assume there exists an orthonormal basis $\widehat{V}_{m} \in$ $\mathbb{C}^{n \times m}$ and a perturbation $\widetilde{U}_{m} \in \mathbb{C}^{n \times m}$, which is sufficiently small in norm (i.e., there exists a moderate constant $C_{3}$ with $\left\|\widetilde{U}_{m}\right\|_{2} \leq C_{3} \varepsilon$ ), with

$$
H_{m}=\widehat{V}_{m}^{*} A \widehat{V}_{m}+\widetilde{U}_{m}
$$

With assumption (A.2) and basic properties of the numerical range we obtain

$$
\mathrm{W}\left(H_{m}\right) \subseteq \mathrm{W}\left(\widehat{V}_{m}^{*} A \widehat{V}_{m}\right)+\mathrm{W}\left(\widetilde{U}_{m}\right)
$$

Similar to (A.1) we obtain

$$
\mathrm{W}\left(\widehat{V}_{m}^{*} A \widehat{V}_{m}\right) \subseteq \mathrm{W}(A)
$$

Then, we combine (A.3) and (A.4) and make use of $\left\|\widetilde{U}_{m}\right\|_{2} \leq C_{3} \varepsilon$ to obtain

$$
\mathrm{W}\left(H_{m}\right) \subseteq U_{C_{3} \varepsilon}(\mathrm{W}(A))
$$

with $U_{C_{3} \varepsilon}(\mathrm{W}(A))$ being the neighborhood of $\mathrm{W}(A)$ with a distance $C_{3} \varepsilon$.

In [50, Theorem 5], the existence of the representation (A.2) is proven for the Lanczos method with a sufficiently small constant $C_{3}$ and the assumption that the Krylov basis is semiorthogonal.

For the general case of the Arnoldi method the representation (A.2) can be derived using (2.6), (2.7a) and an additional condition on the level of orthogonality of the Krylov basis, e.g., assuming that an orthonormal basis $\widehat{V}_{m}$ exists for which $\| \widehat{V}_{m}-$ $V_{m} \|_{2}$ is small enough (see also [7, Theorem 2.1] and references therein). 


\section{Appendix B: Some properties of divided differences}

Proof of Proposition 1 For $p \in \mathbb{N}_{0}$ and any $A \in \mathbb{C}^{m \times m}, w \in \mathbb{C}^{m}$, from the series representation (2.2) we obtain

$\int_{0}^{t} s^{p} \varphi_{p}(s A) w \mathrm{~d} s=\int_{0}^{t}\left(\sum_{k=0}^{\infty} \frac{s^{k+p} A^{k} w}{(k+p) !}\right) \mathrm{d} s=\sum_{k=0}^{\infty} \frac{t^{k+p+1} A^{k} w}{(k+p+1) !}=t^{p+1} \varphi_{p+1}(t A) w$.

This identity carries over to divided differences in the following way. Let

$$
\Theta_{m}=\left(\begin{array}{cccc}
\lambda_{1} & & & \\
1 & \lambda_{2} & & \\
& \ddots & \ddots & \\
& & 1 & \lambda_{m}
\end{array}\right) \in \mathbb{C}^{m \times m} .
$$

As a consequence of the Opitz formula, see [42] and remarks in [4, Proposition 25], we have

$$
\left(\varphi_{p}\right)_{t}\left[\lambda_{1}, \ldots, \lambda_{m}\right]=e_{m}^{*} \varphi_{p}\left(t \Theta_{m}\right) e_{1} .
$$

Using (B.1) and (B.2), we obtain

$$
\begin{aligned}
\int_{0}^{t} s^{p}\left(\varphi_{p}\right)_{s}\left[\lambda_{1}, \ldots, \lambda_{m}\right] \mathrm{d} s & =e_{m}^{*} \int_{0}^{t} s^{p} \varphi_{p}\left(s \Theta_{m}\right) e_{1} \mathrm{~d} s=e_{m}^{*} t^{p+1} \varphi_{p+1}\left(t \Theta_{m}\right) e_{1} \\
& =t^{p+1}\left(\varphi_{p+1}\right)_{t}\left[\lambda_{1}, \ldots, \lambda_{m}\right],
\end{aligned}
$$

which completes the proof.

Remark 11 We will make use of the following integral representation for divided differences, the so-called Hermite-Genocchi formula, [24, (B.25)]. With the differential operator $\left(D^{(m-1)} f_{t}\right)(\lambda)=\frac{\mathrm{d}^{m-1}}{\mathrm{~d} \lambda^{m-1}} f(t \lambda)$,

$$
\begin{aligned}
f_{t}\left[\lambda_{1}, \ldots, \lambda_{m}\right] & =\int_{\left[\lambda_{1}, \ldots, \lambda_{m}\right]} D^{(m-1)} f_{t} \\
& =\int_{0}^{1} \int_{0}^{s_{1}} \cdots \int_{0}^{s_{m-2}} D^{(m-1)} f\left(\lambda_{1}+\sum_{j=1}^{m-1} s_{j}\left(\lambda_{j+1}-\lambda_{j}\right)\right) \mathrm{d} s_{m-1} \ldots \mathrm{d} s_{2} \mathrm{~d} s_{1} .
\end{aligned}
$$

Proof of Proposition 2 Applying (B.3) to the exponential function gives

$$
\begin{aligned}
\left|\exp _{t}\left[\lambda_{1}, \ldots, \lambda_{k}\right]\right| & \leq \int_{0}^{1} \int_{0}^{s_{1}} \ldots \int_{0}^{s_{k-2}} t^{k-1}\left|\exp \left(\lambda_{1}+\sum_{j=1}^{k-1} s_{j}\left(\lambda_{j+1}-\lambda_{j}\right)\right)\right| \mathrm{d} s_{k-1} \ldots \mathrm{d} s_{2} \mathrm{~d} s_{1} \\
& =\int_{0}^{1} \int_{0}^{s_{1}} \ldots \int_{0}^{s_{k-2}} t^{k-1} \exp \left(\xi_{1}+\sum_{j=1}^{k-1} s_{j}\left(\xi_{j+1}-\xi_{j}\right)\right) \mathrm{d} s_{k-1} \ldots \mathrm{d} s_{2} \mathrm{~d} s_{1} \\
& =\exp _{t}\left[\xi_{1}, \ldots, \xi_{k}\right],
\end{aligned}
$$

which completes the proof. 
Proof of Proposition 3 We use (B.3) to obtain

$$
\begin{aligned}
\exp _{t}\left[\lambda_{1}, \ldots, \lambda_{k}\right]= & \int_{0}^{1} \int_{0}^{s_{1}} \ldots \int_{0}^{s_{k-2}} t^{k-1} \exp \left(t\left(\lambda_{1}+\sum_{j=1}^{k-1} s_{j}\left(\lambda_{j+1}-\lambda_{j}\right)\right)\right) \mathrm{d} s_{k-1} \ldots \mathrm{d} s_{2} \mathrm{~d} s_{1} \\
= & \int_{0}^{1} \int_{0}^{s_{1}} \ldots \int_{0}^{s_{k-2}} t^{k-1} \\
& \cdot\left[\cos \left(t\left(\eta_{1}+\sum_{j=1}^{k-1} s_{j}\left(\eta_{j+1}-\eta_{j}\right)\right)\right)+\mathrm{i} \sin \left(t\left(\eta_{1}+\sum_{j=1}^{k-1} s_{j}\left(\eta_{j+1}-\eta_{j}\right)\right)\right)\right] \\
& \cdot \exp \left(t\left(\xi_{1}+\sum_{j=1}^{k-1} s_{j}\left(\xi_{j+1}-\xi_{j}\right)\right)\right) \mathrm{d} s_{k-1} \ldots \mathrm{d} s_{2} \mathrm{~d} s_{1} \\
= & (\cos (t x)+\mathrm{i} \sin (t y)) \exp _{t}\left[\xi_{1}, \ldots, \xi_{k}\right] \quad \text { for certain } x, y \in \operatorname{Conv}\left(\left\{\eta_{1}, \ldots, \eta_{k}\right\}\right) .
\end{aligned}
$$

Here, in the last step, we have used the Mean Value Theorem for the integral. In this way we end up with the estimate

$$
\left|\exp _{t}\left[\lambda_{1}, \ldots, \lambda_{m}\right]\right|=|\cos (t x)+\mathrm{i} \sin (t y)| \cdot \exp _{t}\left[\xi_{1}, \ldots, \xi_{m}\right] .
$$

With $|t x|,|t y| \leq \tilde{\eta}_{t}<\pi / 2$ we obtain

$$
\cos \left(\tilde{\eta}_{t}\right) \leq \cos (t x) \leq|\cos (t x)+\mathrm{i} \sin (t y)|,
$$

which completes the proof.

\section{Appendix C: A new asymptotic expansion of divided differences}

Our goal is to derive an asymptotic expansion for $\left|\exp _{t}\left[\lambda_{1}, \ldots, \lambda_{m}\right]\right|$, see Theorem 5 at the end of this section.

Let $\lambda_{1}, \ldots, \lambda_{m} \in \mathbb{C}$. We use the shortcut $\kappa_{k}$ for the divided differences of power functions,

$$
\kappa_{k}=(\cdot)^{m-1+k}\left[\lambda_{1}, \ldots, \lambda_{m}\right] \text { for } k \in \mathbb{N}_{0},
$$

where $(\cdot)^{j}: z \mapsto z^{j}$ for $j \in \mathbb{N}_{0}$. Note that

$$
(\cdot)^{j}\left[\lambda_{1}, \ldots, \lambda_{m}\right]=0 \text { for } j=0, \ldots, m-2 .
$$

With the notation (C.1) and the series representation of the exponential function we obtain

$$
\begin{aligned}
\exp _{t}\left[\lambda_{1}, \ldots, \lambda_{m}\right] & =\sum_{j=0}^{\infty} \frac{t^{j}(\cdot)^{j}\left[\lambda_{1}, \ldots, \lambda_{m}\right]}{j !}=t^{m-1} \sum_{k=0}^{\infty} \frac{t^{k} \kappa_{k}}{(m-1+k) !}(\mathrm{C} \\
& =\frac{t^{m-1}}{(m-1) !}+\mathscr{O}\left(t^{m}\right) \text { for } t \rightarrow 0
\end{aligned}
$$


We also introduce the notation

$$
S_{l}=\sum_{j=1}^{m} \lambda_{j}^{l}, \quad l \in \mathbb{N} .
$$

For $\kappa_{0}, \kappa_{1}$, and $\kappa_{2}$, we obtain the following formula.

Proposition 6 For $\kappa_{k}$ introduced in (C.1) we have

$$
\kappa_{0}=1, \quad \kappa_{1}=S_{1}, \quad \kappa_{2}=\left(S_{1}^{2}+S_{2}\right) / 2 .
$$

Proof This follows from [4, (27)].

To simplify the notation, we write

$$
f(t)=\left|\exp _{t}\left[\lambda_{1}, \ldots, \lambda_{m}\right]\right| .
$$

The following asymptotic expansion of $f(t)$ for $t \rightarrow 0$ is motivated by the concept of effective order. The effective order of the function $f(t)$ can be understood as the slope of the double-logarithmic function

$$
\ln \left(f\left(\mathrm{e}^{\tau}\right)\right) \quad \text { with } \tau=\ln t, \quad \text { and with derivative } \frac{f^{\prime}\left(\mathrm{e}^{\tau}\right) \mathrm{e}^{\tau}}{f\left(\mathrm{e}^{\tau}\right)} .
$$

We denote the effective order by

$$
\begin{aligned}
\rho(t) & =\frac{f^{\prime}(t) t}{f(t)}, \\
\text { satisfying } \rho(t) / t & =(\log (f(t)))^{\prime} .
\end{aligned}
$$

We now analyze the divided differences close to an asymptotic regime under the assumption $f(t)>0$, which holds for sufficiently small $t>0$. The effective order $\rho(t)$ is then well-defined by (C.4a). The following expansion (C.5) for $\rho(t)$ is to be considered in an asymptotic sense for $t \rightarrow 0$; convergence of the series is not an issue here.

We make the ansatz

$$
\rho(t)=\sum_{k=0}^{\infty} \rho_{k} t^{k}
$$

Using (C.5) in (C.4b), we obtain

$$
\begin{aligned}
\frac{\rho(t)}{t}=\left(\rho_{0} \log (t)+\sum_{k=1}^{\infty} \rho_{k} t^{k} / k\right)^{\prime} & =(\log (f(t)))^{\prime} \\
c \exp \left(\rho_{0} \log (t)+\sum_{k=1}^{\infty} \rho_{k} t^{k} / k\right) & =f(t) \\
c t^{\rho_{0}} \exp \left(\sum_{k=1}^{\infty} \rho_{k} t^{k} / k\right) & =f(t)
\end{aligned}
$$


From (C.2b), we see that $c=1 /(m-1)$ ! and $\rho_{0}=m-1$, whence

$$
\rho(t)=m-1+\sum_{k=1}^{\infty} \rho_{k} t^{k}
$$

and for sufficiently small $t$,

$$
f(t)=\left|\exp _{t}\left[\lambda_{1}, \ldots, \lambda_{m}\right]\right|=\frac{t^{m-1}}{(m-1) !} \exp \left(\sum_{k=1}^{\infty} \rho_{k} t^{k} / k\right) .
$$

We aim for deriving a formula for the coefficients $\rho_{k}$. To avoid the square roots, we choose $q(t)=f(t)^{2}$, such that $f^{\prime}(t)=q^{\prime}(t) /\left(2 q(t)^{1 / 2}\right)$. Due to (C.4a) the effective order $\rho(t)$ satisfies

$$
q(t) \rho(t)=q^{\prime}(t) t / 2 .
$$

We proceed by rewriting $q(t)$ and $q^{\prime}(t)$ to obtain a formulation for $\rho_{k}(k \geq 1)$ via (C.8). From (C.2a),

$$
q(t)=\left|\exp _{t}\left[\lambda_{1}, \ldots, \lambda_{m}\right]\right|^{2}=t^{2(m-1)}\left(\sum_{k=0}^{\infty} \frac{t^{k} \kappa_{k}}{(m-1+k) !}\right)\left(\sum_{\ell=0}^{\infty} \frac{t^{\ell} \bar{\kappa}_{\ell}}{(m-1+\ell) !}\right) .
$$

The representation of $q(t)$ as well as $t q^{\prime}(t) / 2$ as a Cauchy product can be written in the form

$q(t)=\frac{t^{2(m-1)}}{((m-1) !)^{2}} \sum_{k=0}^{\infty} \alpha_{k} t^{k}, \quad$ and $t q^{\prime}(t) / 2=\frac{t^{2(m-1)}}{((m-1) !)^{2}} \sum_{k=0}^{\infty}((m-1)+k / 2) \alpha_{k} t^{k}$,

with coefficients $\alpha_{k}$ given by

$$
\alpha_{0}=1, \quad \text { and } \quad \alpha_{k}=\sum_{j=0}^{k} \frac{((m-1) !)^{2} \kappa_{j} \bar{\kappa}_{k-j}}{(m-1+j) !(m-1+k-j) !} \text { for } k \in \mathbb{N} .
$$

With $\kappa_{0}=1$ (see Proposition 6), this can be written as

$$
\alpha_{k}=\frac{2(m-1) ! \operatorname{Re}\left(\kappa_{k}\right)}{(m-1+k) !}+\sum_{j=1}^{k-1} \frac{((m-1) !)^{2} \kappa_{j} \bar{\kappa}_{k-j}}{(m-1+j) !(m-1+k-j) !} \quad \text { for } k \in \mathbb{N} \text {. }
$$

Furthermore, from (C.6) and (C.9), we obtain a representation of $q(t) \rho(t)$ in form of a Cauchy product,

$$
q(t) \rho(t)=\frac{t^{2(m-1)}}{((m-1) !)^{2}} \sum_{k=0}^{\infty} \theta_{k} t^{k}, \quad \text { with } \theta_{k}=\sum_{j=0}^{k-1} \alpha_{j} \rho_{k-j}+(m-1) \alpha_{k}, \quad k \in \mathbb{N}_{0} .
$$

We remark that (C.11) only holds for $t$ small enough. With $\alpha_{0}=1$, in (C.11), we have

$$
\theta_{0}=m-1, \quad \text { and } \quad \theta_{k}=\rho_{k}+\sum_{j=1}^{k-1} \alpha_{j} \rho_{k-j}+(m-1) \alpha_{k}, \quad k \in \mathbb{N} .
$$


For the implicit (C.8), we combine (C.9) and (C.11) to obtain

$$
\sum_{k=0}^{\infty} \theta_{k} t^{k}=\sum_{k=0}^{\infty}(m-1+k / 2) \alpha_{k} t^{k}
$$

Comparing coefficients of $t^{k}$ in (C.13) and using (C.12) we conclude

$$
\theta_{k}=(m-1+k / 2) \alpha_{k}, \quad \text { and } \quad \rho_{k}=\frac{k \alpha_{k}}{2}-\sum_{l=1}^{k} \alpha_{l} \rho_{k-l}, \quad k \geq 1 .
$$

From (C.14), we obtain a recursion for the coefficients $\rho_{k}$ in the expansion (C.6) which can be resolved using (C.1) and (C.10).

We now evaluate the lower coefficients of $\rho(t)$. For $\alpha_{1}$ and $\alpha_{2}$, using Proposition 6 in (C.10) gives

$\alpha_{1}=\frac{2 \operatorname{Re}\left(\kappa_{1}\right)}{m}=\frac{2 \operatorname{Re}\left(S_{1}\right)}{m}$, and $\alpha_{2}=\frac{\left|\kappa_{1}\right|^{2}}{m^{2}}+\frac{2 \operatorname{Re}\left(\kappa_{2}\right)}{m(m+1)}=\frac{\left|S_{1}\right|^{2}}{m^{2}}+\frac{\operatorname{Re}\left(S_{1}^{2}+S_{2}\right)}{m(m+1)}$,

with $S_{1}, S_{2}$ according to definition (C.3) From the recursion in (C.14), we have

$$
\rho_{1}=\frac{\alpha_{1}}{2}, \quad \rho_{2}=\frac{1}{2}\left(2 \alpha_{2}-\alpha_{1}^{2}\right),
$$

and combining (C.15) with (C.16), we eventually obtain

$$
\begin{aligned}
& \rho_{1}=\frac{\operatorname{Re}\left(S_{1}\right)}{m}, \\
& \rho_{2}=\frac{\left|S_{1}\right|^{2}}{m^{2}}+\frac{\operatorname{Re}\left(S_{1}^{2}+S_{2}\right)}{m(m+1)}-\frac{2 \operatorname{Re}\left(S_{1}\right)^{2}}{m^{2}}=\frac{\operatorname{Im}\left(S_{1}\right)^{2}-\operatorname{Re}\left(S_{1}\right)^{2}}{m^{2}}+\frac{\operatorname{Re}\left(S_{1}^{2}+S_{2}\right)}{m(m+1)} .
\end{aligned}
$$

To study the influence of the real and imaginary parts of the nodes $\lambda_{j}=\xi_{j}+\mathrm{i} \eta_{j}$, we introduce the notation

$$
S_{l k}=\sum_{j=1}^{m} \xi_{j}^{l} \eta_{j}^{k}, \quad l, k \in \mathbb{N}_{0} .
$$

Basic computations, mostly binomial sums in (C.3), show

$$
S_{1}=S_{10}+\mathrm{i} S_{01}, \quad S_{2}=S_{20}+2 \mathrm{i} S_{11}-S_{02}, \quad \text { and } S_{1}^{2}=S_{10}^{2}+\mathrm{i} S_{10} S_{01}-S_{01}^{2},
$$

and

$$
\operatorname{Im}\left(S_{1}\right)=S_{01}, \quad \operatorname{Re}\left(S_{1}\right)=S_{10}, \quad \operatorname{Re}\left(S_{2}\right)=S_{20}-S_{02}, \quad \text { and } \operatorname{Re}\left(S_{1}^{2}\right)=S_{10}^{2}-S_{01}^{2} .
$$

Combining (C.17) with (C.19) gives

$$
\rho_{1}=\frac{S_{10}}{m}, \quad \text { and } \rho_{2}=\frac{S_{01}^{2}-S_{10}^{2}}{m^{2}(m+1)}+\frac{S_{20}-S_{02}}{m(m+1)} .
$$

After all these technicalities, we arrive at the following asymptotic expansion.

Theorem 5 Assume that for $\lambda_{j}=\xi_{j}+\mathrm{i} \eta_{j}$ at least one of the sequences $\left\{\xi_{j}\right\}_{j=1}^{m}$ and $\left\{\eta_{j}\right\}_{j=1}^{m}$ is not constant, and $\xi_{j} \leq 0$ for $j=1, \ldots$, m. Let $\operatorname{avg}(\xi)=\sum_{j=1}^{m} \xi_{j} / m$ be 
the average and $\operatorname{var}(\xi)=\sum_{j=1}^{m}\left(\xi_{j}-\operatorname{avg}(\xi)\right)^{2} / m$ be the variance of $\left\{\xi_{1}, \ldots, \xi_{m}\right\}$, and $\operatorname{var}(\eta)$ the variance of $\left\{\eta_{1}, \ldots, \eta_{m}\right\}$. Then,

1.

$$
\left|\exp _{t}\left[\lambda_{1}, \ldots, \lambda_{m}\right]\right|=\frac{t^{m-1}}{(m-1) !} \exp \left(\rho_{1} t+\rho_{2} t^{2} / 2+\mathscr{O}\left(t^{3}\right)\right) \text { for } t \rightarrow 0,
$$

with

$$
\rho_{1}=\operatorname{avg}(\xi), \quad \rho_{2}=\frac{\operatorname{var}(\xi)-\operatorname{var}(\eta)}{m+1},
$$

and either $\rho_{1} \neq 0$ or $\rho_{2} \neq 0$.

2. The derivative of the effective order $\rho(t)($ see $(C .4 a))$ satisfies $\rho^{\prime}(t)=\rho_{1}+\rho_{2} t+$ $\mathscr{O}\left(t^{2}\right)$ for $t \rightarrow 0$, and

$$
\rho^{\prime}(0+)<0
$$

Proof We use the expansion (C.7) for sufficiently small $t$. For the variance, we obtain

$$
\operatorname{var}(\xi)=\frac{1}{m} \sum_{j=1}^{m}\left(\xi_{j}-\operatorname{avg}(\xi)\right)^{2}=\frac{1}{m}\left(\sum_{j=1}^{m} \xi_{j}^{2}-\frac{1}{m}\left(\sum_{j=1}^{m} \xi_{j}\right)^{2}\right) .
$$

The first coefficients $\rho_{1}$ and $\rho_{2}$ are given in (C.20). With the notation from (C.18) we observe $\operatorname{avg}(\xi)=S_{10} / m$ (for the average $\left.\operatorname{avg}(\xi)\right)$ and $\operatorname{var}(\xi)=\left(S_{20}-S_{10}^{2} / m\right) / m$, $\operatorname{var}(\eta)=\left(S_{02}-S_{01}^{2} / m\right) / m$ (for the variance $\operatorname{var}(\xi)$ and $\operatorname{var}(\eta)$, respectively), whence

$$
\rho_{1}=\operatorname{avg}(\xi), \quad \text { and } \rho_{2}=\frac{\operatorname{var}(\xi)-\operatorname{var}(\eta)}{m+1} .
$$

With $\xi_{1}, \ldots, \xi_{m} \leq 0$ for $j=1, \ldots, m$ we obtain $\rho_{1} \leq 0$ and $\rho_{1}=0$ iff $\xi_{1}, \ldots, \xi_{m}=$ 0 . For the case $\xi_{1}, \ldots, \xi_{m}=0$, we obtain $\operatorname{var}(\xi)=0$ and

$$
\rho_{2}=-\frac{\operatorname{var}(\eta)}{m+1} \leq 0
$$

Here, $\rho_{2}=0$ only in the trivial case with $\xi_{1}, \ldots, \xi_{m}=0$ and a constant sequence $\eta_{1}, \ldots, \eta_{m}$. This proves (a). For the proof of (b) we take the derivative of $\rho(t)$ in an asymptotic sense and make use of $\rho_{1} \leq 0$ and $\rho_{2}<0$ iff $\rho_{1}=0$, see (a).

Funding Open access funding provided by TU Wien (TUW). This work was supported by the Doctoral College TU-D, Technische Universität Wien.

Open Access This article is licensed under a Creative Commons Attribution 4.0 International License, which permits use, sharing, adaptation, distribution and reproduction in any medium or format, as long as you give appropriate credit to the original author(s) and the source, provide a link to the Creative Commons licence, and indicate if changes were made. The images or other third party material in this article are included in the article's Creative Commons licence, unless indicated otherwise in a credit line to the material. If material is not included in the article's Creative Commons licence and your intended use is not permitted by statutory regulation or exceeds the permitted use, you will need to obtain permission directly from the copyright holder. To view a copy of this licence, visit http://creativecommons.org/licenses/by/4.0/. 


\section{References}

1. Afanasjew, M., Eiermann, M., Ernst, O., Güttel, S.: Implementation of a restarted Krylov subspace method for the evaluation of matrix functions. Linear Algebra Appl. 429(10), 2293-2314 (2008). https://doi.org/10.1016/j.laa.2008.06.029

2. Al-Mohy, A., Higham, N.: Computing the action of the matrix exponential, with an application to exponential integrators. SIAM J. Sci. Comput. 33(2), 488-511 (2011). https://doi.org/10.1137/10078 8860

3. Beckermann, B., Reichel, L.: Error estimates and evaluation of matrix functions via the Faber transform. SIAM J. Numer. Anal. 47(5), 3849-3883 (2009). https://doi.org/10.1137/080741744

4. de Boor, C.: Divided differences. Surv. Approx. Theory 1, 46-69 (2005)

5. Botchev, M., Grimm, V., Hochbruck, M.: Residual, restarting and Richardson iteration for the matrix exponential. SIAM J. Sci. Comput. 35(3), A1376-A1397 (2013). https://doi.org/10.1137/110820191

6. Botchev, M., Knizhnerman, L.: ART: Adaptive Residual-time restarting for Krylov subspace matrix exponential evaluations. J. Comput. Appl. Math. https://doi.org/10.1016/j.cam.2019.06.027 (2019)

7. Braconnier, T., Langlois, P., Rioual, J.: The influence of orthogonality on the Arnoldi method. Linear Algebra Appl. 309(1), 307-323 (2000). https://doi.org/10.1016/S0024-3795(99)00100-7

8. Caliari, M., Kandolf, P., Ostermann, A., Rainer, S.: The Leja method revisited: backward error analysis for the matrix exponential. SIAM J. Sci. Comput. 38(3), A1639-A1661 (2016). https://doi.org/10.1137/15M1027620

9. Celledoni, E., Moret, I.: A Krylov projection method for systems of ODEs. Appl. Numer. Math. 24(2), 365-378 (1997). https://doi.org/10.1016/S0168-9274(97)00033-0

10. Diele, F., Moret, I., Ragni, S.: Error estimates for polynomial Krylov approximations to matrix functions. SIAM J. Matrix Anal. Appl. 30(4), 1546-1565 (2009). https://doi.org/10.1137/070688924

11. Druskin, V., Greenbaum, A., Knizhnerman, L.: Using nonorthogonal Lanczos vectors in the computation of matrix functions. SIAM J. Sci. Comput. 19(1), 38-54 (1998). https://doi.org/10.1137/S10648 27596303661

12. Druskin, V., Knizhnerman, L.: Two polynomial methods of calculating functions of symmetric matrices. USSR Comput Math. Math. Phys. 29(6), 112-121 (1989). https://doi.org/10.1016/S00415553(89)80020-5

13. Druskin, V., Knizhnerman, L.: Error bounds in the simple Lanczos procedure for computing functions of symmetric matrices and eigenvalues. Comput. Math. Math. Phys. 31(7), 20-30 (1992)

14. Druskin, V., Knizhnerman, L.: Extended Krylov subspaces: Approximation of the matrix square root and related functions. SIAM. J. Matrix Anal. Appl. 19(3), 755-771 (1998). https://doi.org/10.1137/S0895479895292400

15. Eiermann, M., Ernst, O.: A restarted Krylov subspace method for the evaluation of matrix functions. SIAM J. Numer. Anal. 44, 2481-2504 (2006). https://doi.org/10.1137/050633846

16. Eiermann, M., Ernst, O., Güttel, S.: Deflated restarting for matrix functions. SIAM J. Matrix Anal. Appl. 32(2), 621-641 (2011). https://doi.org/10.1137/090774665

17. van den Eshof, J., Hochbruck, M.: Preconditioning Lanczos approximations to the matrix exponential. SIAM J. Sci. Comput. 27(4), 1438-1457 (2006). https://doi.org/10.1137/040605461

18. Friesner, R., Tuckerman, L., Dornblaser, B., Russo, T.: A method for exponential propagation of large systems of stiff nonlinear differential equations. J. Sci. Comput. 4(4), 327-354 (1989). https://doi.org/10.1007/BF01060992

19. Frommer, A., Güttel, S., Schweitzer, M.: Efficient and stable Arnoldi restarts for matrix functions based on quadrature. SIAM J. Matrix Anal. Appl. 35(2), 661-683 (2014). https://doi.org/10.1137/ $13093491 \mathrm{X}$

20. Gallopoulos, E., Saad, Y.: Efficient solution of parabolic equations by Krylov approximation methods. SIAM J. Sci. Statist. Comput. 13(5), 1236-1264 (1992). https://doi.org/10.1137/0913071

21. Göckler, T., Grimm, V.: Convergence analysis of an extended Krylov subspace method for the approximation of operator functions in exponential integrators. SIAM J. Numer. Anal. 51(4), 2189-2213 (2013). https://doi.org/10.1137/12089226X

22. Güttel, S.: Rational Krylov methods for operator functions. Ph.D. thesis, Technische universität Bergakademie Freiberg, Germany. http://eprints.ma.man.ac.uk/2586/. Dissertation available as MIMS Eprint 2017.39 (2010)

23. Higham, N. Accuracy and Stability of Numerical Algorithms, 2nd edn. Society for Industrial and Applied Mathematics, USA (2002). https://doi.org/10.1137/1.9780898718027 
24. Higham, N.: Functions of matrices. Society for industrial and applied mathematics, philadelphia, PA USA. https://doi.org/10.1137/1.9780898717778 (2008)

25. Hochbruck, M., Hochstenbach, M.: Subspace extraction for matrix functions. Tech. rep., Dept. of Math., Case Western Reserve University. http://na.math.kit.edu/download/papers/funext.pdf (2005)

26. Hochbruck, M., Lubich, C.: On Krylov subspace approximations to the matrix exponential operator. SIAM J. Numer. Anal. 34(5), 1911-1925 (1997). https://doi.org/10.1137/S0036142995280572

27. Hochbruck, M., Ostermann, A.: Exponential integrators. Acta Numerica 19, 209-286 (2010). https://doi.org/10.1017/S0962492910000048

28. Hochbruck, W., Lubich, C., Selhofer, H.: Exponential integrators for large systems of differential equations. SIAM J. Sci. Comput. 19(5), 1552-1574 (1998). https://doi.org/10.1137/S106482759529 5337

29. Iserles, A., Kropielnicka, K., Singh, P.: Compact schemes for laser-matter interaction in schrödinger equation based on effective splittings of Magnus expansion. J. Comput. Phys. Comm. 234, 195-201 (2019). https://doi.org/10.1016/j.cpc.2018.07.010

30. Jawecki, T., Auzinger, W., Koch, O.: Computable upper error bounds for Krylov approximations to matrix exponentials and associated $\varphi$-functions BIT. https://doi.org/10.1007/s10543-019-00771-6 (2019)

31. Jia, Z., Lv, H.: A posteriori error estimates of Krylov subspace approximations to matrix functions. Numer. Algorithms 69(1), 1-28 (2015). https://doi.org/10.1007/s11075-014-9878-0

32. Knizhnerman, L., Simoncini, V.: A new investigation of the extended Krylov subspace method for matrix function evaluations. Numer. Linear Algebra Appl. 17, 615-638 (2010). https://doi.org/10.1002/nla.652

33. Kuleff, A., Breidbach, J., Cederbaum, L.: Multielectron wave-packet propagation: General theory and application. J. Chem. Phys. 123(4), 044111 (2005). https://doi.org/10.1063/1.1961341

34. Lubich, C.: From Quantum to Classical Molecular Dynamics; Reduced Models and Numerical Analysis. Zurich lectures in advanced mathematics. European Math. Soc. zürich (2008)

35. McCurdy, A., Ng, K., Parlett, B.: Accurate computation of divided differences of the exponential function. Math. Comp. 43, 501-528 (1984). https://doi.org/10.2307/2008291

36. Moler, C., Van Loan, C.: Nineteen dubious ways to compute the exponential of a matrix, twenty-five years later. SIAM Rev. 45(1), 3-49 (2003). https://doi.org/10.1137/S00361445024180

37. Moret, I., Novati, P.: An interpolatory approximation of the matrix exponential based on Faber polynomials. J. Comput. Appl. Math. 131(1), 361-380 (2001). https://doi.org/10.1016/S0377-0427 (00)00261-2

38. Moret, I., Novati, P.: RD-rational approximations of the matrix exponential. BIT 44, 595-615 (2004). https://doi.org/10.1023/B:BITN.0000046805.27551.3b

39. Nauts, A., Wyatt, R.: New approach to many-state quantum dynamics: the recursive-residuegeneration method. Phys. Rev. Lett. 51, 2238-2241 (1983). https://doi.org/10.1103/PhysRevLett.51. 2238

40. Niehoff, J.: Projektionsverfahren Zur Approximation Von Matrixfunktionen Mit Anwendungen Auf Die Implementierung Exponentieller Integratoren. Ph.D. thesis, Heinrich-Heine-Universität Düsseldorf (2007)

41. Niesen, J., Wright, W.: Algorithm 919: A Krylov subspace algorithm for evaluating the $\phi$ functions appearing in exponential integrators. ACM Trans. Math. Softw. 38(3), 22:1-22:19 (2012). https://doi.org/10.1145/2168773.2168781

42. Opitz, G.: Steigungsmatrizen. Z. Angew. Math. Mech. 44(S1), T52-T54 (1964). https://doi.org/10. 1002/zamm.19640441321

43. Paige, C.: Error analysis of the Lanczos algorithm for tridiagonalizing a symmetric matrix. IMA J. Appl. Math. 18(3), 341-349 (1976). https://doi.org/10.1093/imamat/18.3.341

44. Park, T., Light, J.: Unitary quantum time evolution by iterative Lanczos reduction. J. Chem. Phys. 85, 5870-5876 (1986). https://doi.org/10.1063/1.451548

45. Parlett, B.: The symmetric eigenvalue problem. Society for industrial and applied mathematics, philadelphia, PA USA. https://doi.org/10.1137/1.9781611971163 (1998)

46. Saad, Y.: Analysis of some Krylov subspace approximations to the matrix exponential operator. SIAM J. Numer. Anal. 29(1), 209-228 (1992). https://doi.org/10.1137/0729014

47. Saad, Y.: Iterative methods for sparse linear systems, 2nd edn. Society for Industrial and Applied Mathematics, Philadelphia, PA USA (2003) 
48. Schweitzer, M.: Restarting and error estimation in polynomial and extended Krylov subspace methods for the approximation of matrix functions. Ph.D. thesis, Bergische Universität Wuppertal, Germany. http://nbn-resolving.de/urn/resolver.pl?urn=urn $\% 3 \mathrm{Anbn} \% 3 \mathrm{Ade} \% 3 \mathrm{Ahbz}$ \%3A468-20160212-112106-7 (2015)

49. Sidje, R.: Expokit: A software package for computing matrix exponentials. ACM Trans. Math. Software 24(1), 130-156 (1998). https://doi.org/10.1145/285861.285868

50. Simon, H.: Analysis of the symmetric Lanczos algorithm with reorthogonalization methods. Linear Algebra Appl. 61, 101-131 (1984). https://doi.org/10.1016/0024-3795(84)90025-9

51. Singh, P.: Sixth-order schemes for laser-matter interaction in the Schrödinger equation. J. Chem. Phys. 150(15), 154111 (2019). https://doi.org/10.1063/1.5065902

52. Stewart, D., Leyk, T.: Error estimates for Krylov subspace approximations of matrix exponentials. J. Comput. Appl. Math. 72(2), 359-369 (1996). https://doi.org/10.1016/0377-0427(96)00006-4

53. Tal-Ezer, H.: On restart and error estimation for Krylov approximation of $W=F(A) V$. SIAM J. Sci. Comput. 29(6), 2426-2441 (2007). https://doi.org/10.1137/040617868

54. Tal-Ezer, H., Kosloff, R.: An accurate and efficient scheme for propagating the time dependent schrödinger equation. J. Chem. Phys. 81(9), 3967-3971 (1984). https://doi.org/10.1063/1.448136

55. Van Loan, C.: The sensitivity of the matrix exponential. SIAM J. Numer. Anal. 14(6), 971-981 (1977). https://doi.org/10.1137/0714065

56. Wang, H., Ye, Q.: Error bounds for the Krylov subspace methods for computations of matrix exponentials. SIAM J. Matrix Anal. Appl. 38(1), 155-187 (2017). https://doi.org/10.1137/16M1063733

57. Wu, G., Zhang, L., Xu, T.: A framework of the harmonic Arnoldi method for evaluating $\phi$ functions with applications to exponential integrators. Adv. Comput. Math. 42(3), 505-541 (2016). https://doi.org/10.1007/s10444-015-9433-0

58. Zemke, J.: Krylov Subspace Methods in Finite Precision : a Unified Approach. Ph.D. thesis, Technische Universität Hamburg. https://doi.org/10.15480/882.8 (2003)

Publisher's note Springer Nature remains neutral with regard to jurisdictional claims in published maps and institutional affiliations. 